\author{
Federal Reserve Bank of Dallas \\ Globalization and Monetary Policy Institute \\ Working Paper No. 103 \\ http://www.dallasfed.org/assets/documents/institute/wpapers/2011/0103.pdf
}

\title{
Size, Openness, and Macroeconomic Interdependence ${ }^{*}$
}

\author{
Alexander Chudik \\ Federal Reserve Bank of Dallas and CIMF \\ Roland Straub \\ European Central Bank
}

December 2011

\begin{abstract}
The curse of dimensionality, a problem associated with analyzing the interaction of a relatively large number of endogenous macroeconomic variables, is a prevailing issue in the open economy macro literature. The most common practice to mitigate this problem is to apply the so-called Small Open Economy Framework (SOEF). In this paper, we aim to review under which conditions the SOEF is a justifiable approximation and how severe the consequences of violation of key conditions might be. Thereby, we use a multicountry general equilibrium model as a laboratory. First, we derive the conditions that ensure the existence of the equilibrium and study the properties of the equilibrium using large $N$ asymptotics. Second, we show that the SOEF is a valid approximation only for economies (i) that have a diversified foreign trade structure and if (ii) there is no globally dominant economy in the system. Third, we illustrate that macroeconomic interdependence is primarily related to the degree of trade diversification, and not to the extent of trade openness. Furthermore, we provide some evidence on the pattern of global macroeconomic interdependence by calculating probability impulse response functions in our calibrated multicountry model using data for 153 economies.
\end{abstract}

JEL codes: F41

\footnotetext{
Alexander Chudik, Federal Reserve Bank of Dallas, 2200 N. Pearl Street, Dallas, TX 75201. 214-922-5769. alexander.chudik@dal.frb.org. Roland Straub, European Central Bank, Kaiserstrasse 29, 60311 Frankfurt am Main, Germany. +49- 69-1344-0. roland.straub@ecb.int. Earlier version of the paper was circulated under the title: "Equlibrium of a Large Multicountry DSGE Model." We are indebted to Prof. Hashem Pesaran for his support and many useful discussions. We would like to thank also Prof. Seppo Honkapohja and Dr. Rupert Gatti for their guidance during the earlier stages of this research; and Kevin Sheedy and Dennis Novy for helpful discussions. The views in this paper are those of the authors and do not necessarily reflect the views of the European Central Bank, the Federal Reserve Bank of Dallas or the Federal Reserve System.
} 


\section{Introduction}

What determines the impact of foreign shocks on the domestic business cycle? What are the features that drive macroeconomic interdependence? These questions remain of high importance in an increasingly open and globalized world. The analysis of macroeconomic interdependence, however, has been so far constrained in several dimensions in both, the empirical and the theoretical literature.

In the empirical literature, the difficulty in analyzing macroeconomic interdependence starts by recognizing the "curse of dimensionality" that is associated with estimating an unrestricted system featuring a relatively large number of endogenous macroeconomic variables. ${ }^{1}$ There are two existing approaches to mitigate the so-called curse of dimensionality in the literature: $i$ ) shrinkage of the parameter space, and $i$ ) shrinkage of data. Bayesian estimation, which circumvent the dimensionality problem via imposition of priors on the parameters of the model, is an example of the shrinkage of the parameter space. ${ }^{2}$ Much more common practise to deal with the high dimensionality problem in the open economy macroeconomic literature is the second approach, shrinkage of data. Under this approach, the rest of the world is typically approximated with one representative economy, which is constructed as the cross-sectional (trade-)weighted average of foreign economies.

A rather informal justification given in the theoretical literature for the latter choice is to refer to the home economy as being small and open. The two country setup, home country and exogenous foreign country, is indeed the basis of a so called 'small open economy' framework (SOEF) in the theoretical open economy macroeconomic literature (see for instance Schmitt-Grohe and Uribe, 2003). There are two main assumptions in SOEF - that the economy is sufficiently small to have negligible impact on foreign economies (i.e. exogeneity of foreign variables) and that the rest of the world can be approximated by one representative economy constructed as a cross section average of foreign economies. The former assumption is a textbook explanation of a small open economy, and this assumption alone does not help to overcome the dimensionality problem. In fact, from en empirical standpoint, whether some of the variables are treated as endogenous or exogenous does not pose major obstacles for estimation and therefore the former assumption could be easily relaxed. The latter assumption, although rarely discussed in the literature, deals with the dimensionality problem. Unlike in the empirical literature, the main reason for these simplifying modeling choices in the theoretical models seems to be analytical tractability. Naturally, SOEF makes very strong assumptions on the degree of macroeconomic interdependence and the role of idiosyncratic shocks. Ceteris paribus, a rise in trade openness is associated with an increased macroeconomic interdependence. Although SOEF is widespread, no research has yet formally investigated the conditions under which SOEF is a justifiable approximation. We believe this constitutes a major deficiency that needs to be addressed.

Under which conditions is the SOEF justified? What is the relationship between openness, the size of the economy and the degree of macroeconomic interdependence? In this paper, we answer these questions by examining the properties of the equilibrium of an $N$-country open economy model as $N$ becomes large. Our

\footnotetext{
${ }^{1}$ Even with as few as 4 macroeconomic variables per country, it is not possible to reliably estimate an unrestricted VAR featuring more than 2 economies due to the size of typical macroeconomic datasets.

${ }^{2}$ See for example Canova and Ciccarelli (2004) or Canova and Ciccarelli (2009) for a Bayesian multi-counry VAR models with an application to G7 economies. Priors could be derived from theoretical model, as discussed in Del Negro and Schorfheide (2004).
} 
theoretical set up draws on some recent contribution of open economy general equilibrium models as discussed in Benigno and Benigno (2003), Chari, Kehoe, and McGrattan (2002), Corsetti and Pesenti (2001), Gali and Monacelli (2005), Kollmann (2001), and Schmitt-Grohe and Uribe (2003) to mention just a few. These theoretical models feature $N \geq 2$ endogenously related economies or the assumption that one of the economies under consideration is small. ${ }^{3}$ In our set up, the properties of the model are analyzed as the number of countries, $N$, becomes large. First, we show the existence of a well-defined equilibrium in a $N$-country model, and derive the conditions that need to be fulfilled. Second, we apply large $N$ asymptotics to simplify the equilibrium solution of the model.

Indeed large $N$ asymptotics, which are commonly used in the panel data literature but rarely in theoretical macro literature, turn out to be very useful in simplifying the complex equilibrium solution, even under a general pattern of weak cross section dependence of all idiosyncratic shocks to individual economies. ${ }^{4}$ There are three considerable advantages of this type of restrictions, which bind only in the limit: ${ }^{5}(i)$ they can significantly simplify the asymptotic analysis of the equilibrium, (ii) they are quite general in a sense that they do not depend jointly on every individual assumption of the developed model, but are valid for a wider class of open economy models, and ( iii) they allow us to formally define various concepts such as that of 'small' economy, 'negligible impact', or the notions of local and global dominance. As a result, large $N$ asymptotics allows us to study how restrictions on the parameters of the model (namely those defining steady-state bilateral foreign trade share matrix) affect the equilibrium solution, and to assess, thereby, the properties of the dynamic equilibrium under various scenarios about the size and openness of individual economies.

Note that the model provides a laboratory to evaluate the validity of the SOEF that are undertaken in empirical exercises. The aim of the paper, however, is not only to review under which conditions the SOEF is a justifiable approximation but rather to assess how severe the violation of the conditions might be. Interestingly, the analysis of the SOEF assumptions provide some interesting insights into the relationship between macroeconomic interdependence and openness. We show that both $(i)$ the underlying violation of the SOEF and $(i i)$ the relationship between openness and macroeconomic interdependence depend whether one country is being globally dominant or alternatively by the existence of dominance by some countries at the local level.

The results presented in the paper indicate that the degree of macroeconomic interdependence is not necessarily connected to the notion of trade openness as usually contemplated. In fact, an increase in openness could well lead to a decreased dependence of home economy on foreign shocks. Furthermore, the assumption that an economy is small and open does not justify approximating the rest of the world with one representative economy, constructed by a cross-sectional (trade-)weighted average of the rest of the world, nor justifies the asymptotic exogeneity of foreign variables. Our findings suggests that the degree of trade diversification is a key

\footnotetext{
${ }^{3}$ One exception is model of Gali and Monacelli (2005), where world economy consists of unit mass of countries.

${ }^{4}$ Concept of weak and strong cross-sectional dependence, formally defined in Chudik, Pesaran, and Tosetti (2010), will be applied to the multicountry open economy model.

${ }^{5}$ Restrictions that bind only in the limit as the number of variables approaches infinity were proposed by Chudik and Pesaran (2010) who consider econometric analysis of infinite-dimensional VAR models. This paper apply limiting restrictions on the bilateral foreign trade flows and study the properties of equilibrium as the number of countries becomes large.
} 
parameter in the chosen set up. We identify an intuitive analogy with the asset market literature (Chamberlain, 1983, and Chamberlain and Rothschild, 1983). It is well known that only systemic risk has a bearing on effectively diversified portfolios. Similar reasoning applies here. If a country diversifies its foreign trade and no economy in the world is globally dominant, then (asymptotically as $N \rightarrow$ $\infty)$ an equilibrium solution for domestic endogenous variables does not depend on the idiosyncratic shocks to foreign economies, only home idiosyncratic shocks and common factors (akin to a systemic risk in asset price models) are important. Since it is easier for larger economies to have a diversified foreign trade structure - as the empirical success of gravity models of international trade suggest (Baier and Bergstrand, 2001; or Anderson and Wincoop, 2003) - SOEF seems to be more suitable, somewhat paradoxically, for larger economies. If foreign trade flows cannot be considered as diversified, then SOEF cannot be applied and it might well be the case that some (small and open) economies are endogenously related (even as $N \rightarrow \infty)$, even though the impact of each economy on the rest of the world as a whole is negligible. Therefore one suggestion for applied research is to examine foreign trade flows before reaching a decision about the most appropriate modelling choice. Our analysis thus provides a clear implications for applied researcher about how to model domestic and foreign economies outside the limitations of the SOEF.

We also investigate the pattern of macroeconomic interdependence by calculating probability impulse response functions following monetary policy shocks in our multicountry model calibrated using data for 153 economies. Our analysis suggests that while the United States and the euro area are locally dominant for a number of economies, they overall impact on each other via the trade channel might be overestimated.

The remainder of this paper is organized as follows. Section 2 first briefly summarizes the model of the world economy, and then describes the equilibrium of the model for a finite number of countries. Section 3 investigates the equilibrium as the number of countries, $N$, becomes large and analyzes the degree of macroeconomic interdependence under varying cross-country linkages. Some stylized facts and simulation exercises are presented in Section 4. The final section offers some concluding remarks. Detailed description of the model, derivations and other technical details are given in Appendix.

\section{A Multicountry General Equilibrium Model}

In this section, we present our multicountry open economy general equilibrium model that we utilize for our analyses. The model in the literature that is closest to our framework is perhaps that of Gali and Monacelli (2005). An important distinction is, however, that our model is an $N$-country model which $(i)$ allows for general asymmetric trade flows, $(i i)$ permits general pattern of (cross-sectional) dependencies of individual shocks across countries, and (iii) accommodates economies of different sizes. ${ }^{6}$ Note that the focus of the presented model is not its ability to replicate short-run dynamic properties of the data. The choice of simplicity is well justified at this stage by the observation that the main findings depend only on the order of magnitudes of the coefficients in the canonical system characterizing equilibrium.

\footnotetext{
${ }^{6}$ The framework of Gali and Monacelli (2005) is not suitable for investigating interdependence among individual economies because: $i$ ) set of economies is not countable and each economy is of zero measure, $i i)$ by construction, the share of trade between any pair of economies $(i, j)$ on the total foreign trade of economy $i$ (or $j$ ) is equal zero, and $i i i$ ) all idiosyncratic shocks are independently distributed.
} 
Our model reduces to a standard closed economy textbook macroeconomic model for $N=1$. Novelty comes solely from the country dimension. In our set up, the world consists of $N$ countries indexed by $i \in\{1,2, \ldots, N\}$ where country 1 is chosen as a numeraire (without the loss of generality). Each country is populated by three types of agents: population $\mathcal{P}_{i}$ of households, by a unit mass of firms and a central bank. Households within each country are identical. Each household optimizes a felicity function separable in labor $L_{i t}$ and consumption $C_{i t}$ defined as a Cobb-Douglas function of domestic and foreign goods produced all around the world. All goods are tradeable in nature, but the international shipment of goods is costly. A convenient iceberg trade cost structure is assumed where fraction $\tau_{i j}$ of goods 'melts' on the way from source country $j$ to destination country $i$. Firms are monopolistically competitive: they choose domestic prices $P_{i t}^{d}$ to maximize profit, but they ignore their impact on the overall price level. Besides goods markets and labour markets, households also participate in asset markets, which are assumed to be complete. Households in country $i$ receive a common economy-wide wage $W_{i t}$ per one unit of supplied labour. The price of labour is perfectly flexible, but there are nominal rigidities in the goods markets, hence there is a role for monetary policy. The central bank of each country is responsible for conducting monetary policy by setting one-period interest rate $r_{i t}$. Note that the world is allowed to be asymmetric - technology $A_{i t}$, for $i=1,2, . ., N$, is heterogeneous across countries and follows a serially and cross sectionally dependent process. Each economy has its own position in the world, which is related among others to the bilateral trade costs, the amount of labour supplied by the population, and preferences of households for goods produced abroad. The equilibrium of the model is solved approximately using traditional solution concept of log-linearization around the deterministic steadystate.

Finally, a word on notations is in place. In order to make notation as transparent as possible, following conventions are adhered to. Subscript $\ell$ is used to denote a particular firm and/or good. Subscripts $i$ and $j$ are used to denote a particular country, i.e. $i, j \in\{1,2, \ldots, N\}$. Unit mass of firms in country $i$ is $I_{i}, I_{i} \cap I_{j}=\emptyset$ for $i \neq j$. Vectors are denoted by bold lower case letters. Matrices with the exception of few diagonal matrices are represented by bold upper case letters. $\check{\mathbf{x}}$ is diagonal matrix with vector $\mathbf{x}$ on its diagonal. Lower case letters denote logarithms. Table 1 in Appendix summarizes main variables and symbols used.

In the next step, we present the canonical representation of the log-linearized equilibrium of our multi-country model, while detailed description of the model is relegated to Appendix. ${ }^{7}$

2.1. A Canonical Representation of the Equilibrium. In this section, we present the equilibrium of our multi-country open economy model. For brevity, we focus thereby only on the canonical representation of equilibrium while a detailed derivations of the corresponding equilibrium conditions are presented in the Appendix. By presenting only the canonical system of equations, we follow a common strategy in New Keynesian models to reduce the log-linearized system of equations characterizing equilibrium into ( $i$ ) New Keynesian Phillips curves (NKPC), ( $i i)$ dynamic investment-saving (IS) curves and (iii) monetary policy rules. This system of equations features finally three endogenous variables per economy only: output

\footnotetext{
${ }^{7}$ Extensions such as two (tradeable and nontradeable) sector economies, endogenous (timevarying) import shares, more general risk aversion of households, and imperfect international risk sharing of households due to the friction present in the goods markets would not affect the main findings presented in this paper.
} 
gap, interest rates and inflation (for example Gali and Monacelli, 2005, or Chapter 4 of Woodford, 2003). There are two exogenous shocks per economy in our model - a monetary policy shock and a technology shock - and thereby we shall later reduce the system of equations characterizing equilibrium further into $2 N$ equations featuring domestic output gaps and domestic inflation only.

In the next steps, we describe the corresponding solution for our multi-country model. First, as common in the literature the NKPC relates domestic price inflation to variation in the output gap and is described by:

$$
\pi_{i t}^{d}=\beta \mathrm{E}_{t} \pi_{i, t+1}^{d}+\lambda_{i}(1+\varphi) x_{i t}, \text { for } i=1,2, \ldots, N,
$$

where $\pi_{i t}^{d}$ is producer price (PPI) inflation in country $i, \mathrm{E}_{t}$ is expectation operator conditional on available information at the time $t$, constant $\lambda_{i}$ is defined as $\lambda_{i}=$ $\left(1-\beta \delta_{i}\right)\left(1-\delta_{i}\right) / \delta_{i}, \beta$ is the time preference rate and $\delta_{i}$ is the Calvo coefficient capturing the degree of nominal rigidity in the goods markets. $x_{i t}$ is the output gap in country $i$, defined as $x_{i t} \equiv y_{i t}-\bar{y}_{i t}$, where $y_{i t}$ denotes the (log) output, and the potential output $\bar{y}_{i t}$ is the (log) level of output that would prevail if all prices were flexible, and, as it turns out, is given by:

$$
\bar{y}_{i t}=a_{i t}+\vartheta_{1 i}
$$

in which $a_{i t}$ is labour productivity, and the constant term $\vartheta_{1 i}=\left[\ln \chi_{i i}-\ln \psi_{i i}-\mu-\right.$ $\left.(1+\varphi) \ln \mathcal{P}_{i}\right] /(1+\varphi)$ depends on the structural parameters of the model. That is the potential output of the economy depends upon the parameter $\varphi$ capturing the inverse of the labour supply elasticity, $\chi_{i i}$ the steady state share of non-exported output of GDP, $\mu$ the steady-state price markup, $\psi_{i i}$ the home bias in consumption and the population of households $\mathcal{P}_{i}$.

The dynamic IS curve mimics the intertemporal optimization of households described by a standard intertemporal Euler equation by expressing consumption $c_{i t}$ as a function of output gap $x_{i t}$. The intertemporal Euler equation for a households in country $i$ is

$$
c_{i t}=\mathrm{E}_{t} c_{i, t+1}-\left(r_{i t}-\mathrm{E}_{t} \pi_{i, t+1}+\ln \beta\right), \text { for } i=1,2, \ldots, N,
$$

where $r_{i t}$ is one period interest rate policy instrument and $\mathrm{E}_{t} \pi_{i, t+1}$ is expected consumer price $(\mathrm{CPI})$ inflation rate in period $t+1$. In solving consumption $c_{i t}$ as a function of output gap $x_{i t}$, one has to take into account the market clearing condition (world output $=$ world consumption), individual production functions, heterogenous preferences of households over goods produced in foreign countries, etc (see the Appendix for a detailed derivation). This will yield a system of dynamic IS curves in the model conveniently written in the matrix form as

$$
\boldsymbol{\Psi}_{\mathbf{x}_{t}}=\mathrm{E}_{t} \boldsymbol{\Psi}_{\mathbf{x}_{t+1}}-\left(\mathbf{r}_{t}-\mathrm{E}_{t} \boldsymbol{\pi}_{t+1}+\ln \beta \cdot \boldsymbol{\tau}_{N}\right)+\mathrm{E}_{t} \boldsymbol{\Psi} \Delta \mathbf{a}_{t+1}
$$

where $\boldsymbol{\tau}_{N}=(1,1, \ldots, 1)^{\prime}$ is $N$-dimensional vector of ones, $\mathbf{r}_{t}=\left(r_{1 t}, r_{2 t} \ldots, r_{N t}\right)^{\prime}$ is $N$ dimensional vector of policy instruments, similarly $\mathbf{x}_{t}=\left(x_{1 t}, x_{2 t}, \ldots, x_{N t}\right)^{\prime}$ and $\boldsymbol{\pi}_{t}=\left(\pi_{1 t}, \pi_{2 t}, \ldots, \pi_{N t}\right)^{\prime}$ are vectors of output gaps and CPI inflations, respectively, and $\boldsymbol{\Psi}$ is $N \times N$ matrix of the consumption shares of imported goods with its $(i, j)^{t h}$ element, $\psi_{i j}$, representing the share of imported goods from country $j$ to country $i$ on the aggregate consumption of households of country $i$. In our model, $\psi_{i j}$, for $i, j=1,2, \ldots, N$, are parameters of Cobb-Douglas preferences of households over goods produced in foreign economies resulting accordingly to:

$$
\sum_{j=1}^{N} \psi_{i j}=1, \text { for any } i=1,2, \ldots, N \text {. }
$$


In what follows, the matrix $\boldsymbol{\Psi}$ will be key in the asymptotic analysis of the equilibrium. Suppose that productivity processes $a_{i t}$ follow an exogenous $\operatorname{AR}(1)$ processes, $a_{i t}=\varepsilon_{a i t}$, for $i=1,2, . ., N$, where

$$
\varepsilon_{a i t}=\rho_{a i} \varepsilon_{a i, t-1}+\zeta_{a i t}, \text { for } i=1,2, . ., N,
$$

$\rho_{a i}$ is autoregressive coefficient, $\left|\rho_{a i}\right| \leq \rho<1$, and $\zeta_{\text {ait }}$ is exogenous technology shock to country $i$, which is serially uncorrelated, but not necessarily cross sectionally uncorrelated. Rewriting the $\mathrm{AR}(1)$ productivity processes will yield in the matrix form:

$$
\mathrm{E}_{t} \Delta \mathbf{a}_{t+1}=-\left(\mathbf{I}_{N}-\check{\boldsymbol{\rho}}_{a}\right) \varepsilon_{a t},
$$

where $\check{\boldsymbol{\rho}}_{a}$ is diagonal matrix with the vector of autoregressive parameters $\boldsymbol{\rho}_{a}=$ $\left(\rho_{a 1}, \rho_{a 2}, \ldots, \rho_{a N}\right)^{\prime}$ on its diagonal. As a result equation $(2.2)$ reduces to

$$
\boldsymbol{\Psi}_{\mathbf{x}_{t}}=\mathrm{E}_{t} \boldsymbol{\Psi}_{\mathbf{X}_{t+1}}-\left(\mathbf{r}_{t}-\mathrm{E}_{t} \boldsymbol{\pi}_{t+1}+\ln \beta \cdot \boldsymbol{\tau}_{N}\right)-\boldsymbol{\Psi}\left(\mathbf{I}_{N}-\check{\boldsymbol{\rho}}_{a}\right) \boldsymbol{\varepsilon}_{a t},
$$

which is a multicountry version of otherwise standard dynamic IS curves. Monetary policy in county $i$ is conducted by setting one-period risk-free nominal interest rate $r_{i t}$. Following Taylor-type monetary policy rules are assumed.

$$
r_{i t}=\phi_{\pi i} \mathrm{E}_{t} \pi_{i, t+1}+\phi_{\pi^{d} i} \pi_{i t}^{d}+\phi_{x i} x_{i t}-\ln \beta+\varepsilon_{r i t}, \text { for } i=1,2, \ldots, N,
$$

where $x_{i t}$ is an output gap in country $i$ precisely defined above, $\varepsilon_{\text {rit }}$ is possibly persistent and possibly cross-sectionally dependent $\operatorname{AR}(1)$ monetary policy shock,

$$
\varepsilon_{\text {rit }}=\rho_{r i} \varepsilon_{r i, t-1}+\zeta_{r i t}, \text { for } i=1,2 \ldots, N,
$$

in which $\left|\rho_{r i}\right| \leq \rho<1$ and $\boldsymbol{\zeta}_{r t}=\left(\zeta_{r 1 t}, \ldots, \zeta_{r N t}\right)^{\prime}$ is a serially uncorrelated process with zero mean, $\mathrm{E}\left(\boldsymbol{\zeta}_{r t}\right)=\mathbf{0}$. Taylor rule $(2.5)$ postulates that monetary authorities adjust interest rate according to the expected CPI inflation, current output gap and domestic producer price inflation.

We allow innovations $\left\{\zeta_{\text {rit }}\right\}$ and $\left\{\zeta_{\text {ait }}\right\}$ to be cross sectionally correlated, since there is no reason to expect that monetary shocks, or technology shocks are uncorrelated across countries, unless one specifies a structural model of spatial diffusion of technology and monetary policy shocks. We assume that the nonnegative definite covariance matrices $\boldsymbol{\Sigma}_{r}=\mathrm{E}\left(\boldsymbol{\zeta}_{r t} \boldsymbol{\zeta}_{r t}^{\prime}\right)$, and $\boldsymbol{\Sigma}_{a}=\mathrm{E}\left(\boldsymbol{\zeta}_{a t} \boldsymbol{\zeta}_{a t}^{\prime}\right)$ are not diagonal, i.e. shocks could be correlated across countries, but matrices $\boldsymbol{\Sigma}_{r}$ and $\boldsymbol{\Sigma}_{a}$ have bounded eigenvalues in $N$. As shown by Pesaran and Tosetti (2010) this specification is indeed quite general and it includes all commonly used spatial models in the literature, such as spatial autoregressive or spatial moving average processes for example. Coefficients $\left\{\phi_{\pi_{i}}\right\},\left\{\phi_{\pi^{d} i}\right\}$ and $\left\{\phi_{x i}\right\}$ in the monetary policy reaction functions are also assumed to be bounded in $N$ in the asymptotic analysis of the equilibrium in Section 3.

2.2. Existence of the Equilibrium. Substituting the system of monetary policy rules (2.5) into the system of dynamic IS equations (2.4) yields

$$
\left(\boldsymbol{\Psi}+\check{\boldsymbol{\phi}}_{x}\right) \mathbf{x}_{t}=\boldsymbol{\Psi} \mathrm{E}_{t} \mathbf{x}_{t+1}+\left(\mathbf{I}_{N}-\check{\boldsymbol{\phi}}_{\pi}\right) \mathrm{E}_{t} \boldsymbol{\pi}_{t+1}-\check{\boldsymbol{\phi}}_{\pi^{d}} \boldsymbol{\pi}_{t}^{d}-\varepsilon_{r t}-\boldsymbol{\Psi}\left(\mathbf{I}_{N}-\check{\boldsymbol{\rho}}_{a}\right) \varepsilon_{a t},
$$

and, after substituting the solution for relationship between CPI and PPI inflations (A.42) in Appendix,

(2.6) $\left(\boldsymbol{\Xi}+\check{\boldsymbol{\phi}}_{x}\right) \mathbf{x}_{t}=\boldsymbol{\Xi} \mathrm{E}_{t} \mathbf{x}_{t+1}+\left(\mathbf{I}_{N}-\check{\boldsymbol{\phi}}_{\pi}\right) \mathrm{E}_{t} \boldsymbol{\pi}_{t+1}^{d}-\check{\boldsymbol{\phi}}_{\pi^{d}} \boldsymbol{\pi}_{t}^{d}-\boldsymbol{\varepsilon}_{r t}-\boldsymbol{\Xi}\left(\mathbf{I}_{N}-\check{\boldsymbol{\rho}}_{a}\right) \boldsymbol{\varepsilon}_{a t}$,

where

$$
\Xi=\check{\boldsymbol{\phi}}_{\pi} \boldsymbol{\Psi}+\mathbf{I}_{N}-\check{\boldsymbol{\phi}}_{\pi} .
$$

$\check{\phi}_{\pi}, \check{\phi}_{\pi^{d}}$ and $\check{\phi}_{x}$ are diagonal matrices with elements $\left\{\phi_{\pi i}\right\}_{i=1}^{N},\left\{\phi_{\pi^{d} i}\right\}_{i=1}^{N}$ and $\left\{\phi_{x i}\right\}_{i=1}^{N}$ on the diagonal, respectively. 
NKPC (2.1) and the system of equations (2.6) form a canonical representation of the world economy with two endogenous variables per economy, namely output gap and PPI inflation. This system of equations can be re-written more compactly as:

$$
\mathbf{A}_{0} \mathbf{z}_{t}=\mathbf{A}_{1} \mathrm{E}_{t} \mathbf{z}_{t+1}+\mathbf{A}_{2} \varepsilon_{t}
$$

$$
\begin{aligned}
& \text { where } \underset{2 N \times 1}{\mathbf{z}_{t}}=\left(\begin{array}{c}
\boldsymbol{\pi}_{t}^{d} \\
\mathbf{x}_{t}
\end{array}\right), \underset{2 N \times 2 N}{\mathbf{A}_{0}}=\left(\begin{array}{cc}
\mathbf{I}_{N} & -(1+\varphi) \check{\boldsymbol{\lambda}} \\
\check{\boldsymbol{\phi}}_{\pi^{d}} & \boldsymbol{\Xi}+\check{\boldsymbol{\phi}}_{x}
\end{array}\right), \underset{2 N \times 2 N}{\mathbf{A}_{1}}=\left(\begin{array}{cc}
\beta \mathbf{I}_{N} & \mathbf{0} \\
\mathbf{I}_{N}-\check{\boldsymbol{\phi}}_{\pi} & \boldsymbol{\Xi}
\end{array}\right) \text {, } \\
& \underset{2 N \times 2 N}{\mathbf{A}_{2}}=\left(\begin{array}{cc}
\mathbf{0} & \mathbf{0} \\
-\mathbf{I}_{N} & -\boldsymbol{\Xi}\left(\mathbf{I}_{N}-\check{\boldsymbol{\rho}}_{a}\right)
\end{array}\right), \underset{2 N \times 1}{\boldsymbol{\varepsilon}_{t}}=\left(\begin{array}{c}
\boldsymbol{\varepsilon}_{r t} \\
\boldsymbol{\varepsilon}_{a t}
\end{array}\right) .
\end{aligned}
$$

This system of linear rational expectation equations above can be solved by conventional methods, see Binder and Pesaran (1997).

Unique determinate equilibrium exists only if all eigenvalues of $2 N \times 2 N$ matrix $\mathbf{A}=\mathbf{A}_{0}^{-1} \mathbf{A}_{1}$ lie inside the unit circle. Lemma 1 provides sufficient technical conditions for determinacy.

Lemma 1. Suppose $\operatorname{rank}(\boldsymbol{\Psi})=N-1$. Then equilibrium exists and the set of monetary policy rules that deliver determinate equilibrium is non-empty for any $N \in \mathbb{N}$. Sufficient conditions for determinate equilibrium for any natural $N$ are

$$
0 \leq \phi_{\pi i} \leq \frac{1}{1-\psi_{i i}}
$$

$$
\underline{h}>\bar{m},
$$

where

$$
\begin{aligned}
\underline{h} & =\min _{i \in\{1, \ldots, N\}} h_{i}, h_{i} \equiv\left|\phi_{\pi i} \psi_{i i}+1-\phi_{\pi i}+\phi_{x i}+(1+\varphi) \lambda_{i} \phi_{\pi^{d} i}\right|, \bar{\lambda}=\max _{i \in\{1, \ldots, N\}}\left|\lambda_{i}\right|, \\
\bar{m} & =\max _{i \in\{1, \ldots, N\}} m_{i}, m_{i} \equiv\left|\phi_{\pi i}\left(1-\psi_{i i}\right)\right|, \bar{\phi}_{\pi^{d}}=\max _{i \in\{1, \ldots, N\}}\left|\phi_{\pi^{d} i}\right|, v_{1}=\max _{i \in\{1, \ldots, N\}}\left|1-\phi_{\pi_{i}}-\phi_{\pi^{d} i} \beta\right|, \\
v_{2} & =\max _{i \in\{1, \ldots, N\}} \beta\left\{\left|\frac{\phi_{\pi i}\left(1-\psi_{i i}\right)}{\phi_{\pi^{d} i}}\right|+\left|\frac{1-\phi_{\pi_{i}}\left(1-\psi_{i i}\right)+\phi_{x i}}{\phi_{\pi^{d} i}}\right|\right\}, v_{3}=\max _{i \in\{1, \ldots, N\}}\left|1-\phi_{\pi i}\right| .
\end{aligned}
$$

Proof is relegated to Appendix.

It follows from conditions established in Lemma 1 that one example of the monetary policy rules, which deliver determinate equilibrium, is $\phi_{\pi i}$ close to 1 , $\phi_{\pi^{d} i}=\phi_{\pi^{d}}>0$ and $\phi_{x i}=\phi_{x}$ for $i=1,2, \ldots, N$ where $\phi_{x}$ is sufficiently large. Alternatively, when $(1+\varphi) \underline{\lambda}>\beta, \underline{\lambda} \equiv \min _{i \in\{1, \ldots, N\}}\left|\lambda_{i}\right|, \phi_{\pi i}$ close to 1 , and $\phi_{x i}=$ $\phi_{x}>0$ for $i=1,2, \ldots, N$, then $\left\{\phi_{\pi^{d} i}\right\}_{i=1}^{N}$ being large enough is also sufficient for the existence of a unique stable equilibrium.

Rank condition for matrix $\boldsymbol{\Psi}$ in Lemma 1 deserves some comment, since it does not resembles any usual condition in the traditional small open economy models in the literature. This condition is sufficient and necessary for ensuring that no country or group of countries is completely insulated from the rest of the world. Violation of this condition would mean that the world can be divided into at least two set of countries that have no trade connections with each other, in which case equilibrium relative prices would not be well defined. Recall that by construction, rows of the import share matrix $\boldsymbol{\Psi}$ sum to one, see (2.3). Therefore $\boldsymbol{\Psi}$ can never 
have full rank. As it turns out, any lower rank, $N-k$ for any $k>1$, means that world can be divided into $k$ isolated groups of countries (proof is not trivial and it is provided in Appendix). Thus the rank condition in Lemma 1 is not at all restrictive, it only rules out uninteresting cases where some countries are totally isolated from the rest of the world.

THEOREM 1. If the set of monetary policies is such that all eigenvalues of $\mathbf{A}$ lie inside the unit circle and $\operatorname{rank}(\mathbf{\Psi})=N-1$, then the unique equilibrium solution for $2 N \times 1$ dimensional vector of endogenous variables $\mathbf{z}_{t}=\left(\boldsymbol{\pi}_{t}^{d \prime}, \mathbf{x}_{t}^{\prime}\right)^{\prime}$ is

$$
\mathbf{z}_{t}=\mathbf{C} \varepsilon_{t},
$$

where

$$
\mathbf{C}=\sum_{k=0}^{\infty} \mathbf{A}^{k} \mathbf{A}_{2} \mathbf{R}^{k}, \mathbf{A}=\mathbf{A}_{0}^{-1} \mathbf{A}_{1}
$$

matrices $\mathbf{A}_{0}, \mathbf{A}_{1}$, and $\mathbf{A}_{2}$ are defined by (2.7), and diagonal matrix $\mathbf{R}$ is

$$
\underset{2 N \times 2 N}{\mathbf{R}}=\left(\begin{array}{cc}
\check{\boldsymbol{\rho}}_{r} & \mathbf{0} \\
\mathbf{0} & \check{\boldsymbol{\rho}}_{a}
\end{array}\right) .
$$

Theorem 1 states that the equilibrium solution to any endogenous variable of the model (if it exists) is in general a function of all exogenous shocks to the system. The exact relationship between endogenous variables and shocks, as specified by the matrix $\mathbf{C}$ in (2.12) depends jointly on all assumptions of the model. As we shall see below, the equilibrium solution (2.12) will simplify considerably as $N \rightarrow \infty$.

Note that the set of eigenvalues of $\mathbf{A}$ inevitably depend on the import share matrix $\boldsymbol{\Psi}$. The analytical expression for the necessary and sufficient set of monetary policy rules that delivers determinacy in general $N$-country set-up does not seem to be trivial, but Lemma 1 has shown that this set is non-empty for any $N \in \mathbb{N}$. Next section presents several interesting asymptotic results, establishing thereby a link between the degree of openness, the size of the economies and the degree of macroeconomic interdependence.

\section{Defining Macroeconomic Interdependence}

This section analyses the degree of macroeconomic interdependence in our multicountry general equilibrium model under varying assumptions about cross-country linkages. In particular, we discuss the interdependence of the economies if the number of countries becomes large, under three different scenarios: $(i)$ all economies are of similar size and have a diversified trade structure; $(i i)$ some economies have a substantial proportion of trade with large trade partners, i.e. the latter are $l o$ cally dominant but lack global importance; and (iii) some economies account for a considerable fraction of world output and trade, i.e. they are globally dominant.

Before operationalizing the concepts above, a word on notations that we will utilize below is in place. Note that $a_{n}=O\left(b_{n}\right)$ states the deterministic sequence $a_{n}$ is at most of order $b_{n}, a_{n}=o\left(b_{n}\right)$ states that $a_{n}$ is of order less than $b_{n}$. Symbol $\stackrel{q . m}{\rightarrow}$ represents convergence in quadratic mean. $\varrho(\mathbf{A})$ is spectral radius of matrix $\mathbf{A}$. All asymptotics below are carried out under $N \rightarrow \infty$. For example, let $N \rightarrow \infty$ and denote the $N \times N$ matrix of import shares as $\boldsymbol{\Psi}_{N}$, where subscript $N$ denotes the number of economies in the world. Then it is assumed in the following exposition that the limits of individual elements of matrix $\boldsymbol{\Psi}_{N}$ exist, that is

$$
\forall i, j \in \mathbb{N}: \lim _{N \rightarrow \infty} \psi_{N i j}=k_{i j}
$$


We will discuss varying forms of cross-country linkages based on a close examination of the properties of the trade matrix as the number of countries $N \rightarrow \infty$. In what follows, we first define formally the concepts discussed above.

Definition 1 (Diversified trade structure). Country $i \in \mathbb{N}$ is said to have diversified imports if $\lim _{N \rightarrow \infty} \psi_{N} i j=k_{i j}=0$ for any $j \neq i, j \in \mathbb{N}$.

Definition 2 (Local dominance). Country $j \in \mathbb{N}$ is said to be locally dominant if $\exists i \in \mathbb{N}, i \neq j$ such that $\lim _{N \rightarrow \infty} \psi_{N} i j=k_{i j} \neq 0$. In this case, country $j$ is also said to be locally dominant for country $i$.

Definition 3 (Global dominance). Country $j \in \mathbb{N}$ is said to be globally dominant if $\sum_{i=1}^{N} \psi_{N i j} \neq o(N)$.

The definitions are straightforward to interpret. Diversified trade structure implies that as the number of countries becomes large, the weight of each country in the trade matrix converges towards zero. Correspondingly, if a country $j$ is locally dominant for country $i$, the import share $\psi_{N i j}$ between the countries will not converges to zero, as the number of countries becomes large. In a similar vein, if country $j$ is globally dominant, than the column sum of import share matrix for column $j$ increases at the rate $N$. Note also that the output share of a globally dominant economy does not converge to zero, even if the number of countries approaches infinity.

3.1. Diversified Trade Structure. In the first step, we describe formally the assumptions for the determining the equilibrium of multi-country model with diversified trade structure as $N \rightarrow \infty$. The logic behind the assumptions will be discussed in details in the next step.

Assumption A1 (Foreign trade flows - many small economies) Let $\boldsymbol{\psi}_{N,-i} \equiv\left(\psi_{N, i, 1}, \ldots, \psi_{N}, i, i-1,0, \psi_{N, i, i+1}, \ldots, \psi_{N, i, N}\right)^{\prime}$. For any $i \in \mathbb{N},\left\|\boldsymbol{\psi}_{N,-i}\right\|_{r}=$ $O\left(N^{-1}\right)$, where $\|\cdot\|_{r}$ denotes the maximum absolute row-sum matrix norm.

Assumption A2 $\lim _{N \rightarrow \infty} \psi_{N, i i} \equiv k_{i}$, for $i=1,2, \ldots$, where $1-k \geq k_{i} \geq k$ for some constant $k>0$, i.e. share of domestically produced tradeable goods on aggregate consumption does not converge to zero (or one) as the number of countries approaches infinity.

Assumption A3 (Population) Countries have bounded relative population: $\exists M>$ $0: \mathcal{P}_{i} / \mathcal{P}_{j}<M, \forall i, j \in \mathbb{N}$.

Assumption A4 Sequence of import share matrices $\left\{\boldsymbol{\Psi}_{N}\right\}$ is such that $\lim _{N \rightarrow \infty} \sum_{k=1}^{N} \psi_{N k j} \mathcal{P}_{k}$ exists for $\forall j \in \mathbb{N}$.

Assumption A5 Set of monetary policy rules satisfies the following conditions.

i) $\exists N_{0} \in \mathbb{N} ; \forall N>N_{0}$ all eigenvalues of $\mathbf{A}_{N}$ lie inside the unit circle.

ii) $\inf _{i \in \mathbb{N}} h_{i}>\sup _{i \in \mathbb{N}} m_{i}$ for $\forall i \in \mathbb{N}$, where $h_{i}$ and $m_{i}$ are defined in Lemma $1 .^{8}$

iii) Any point on the unit circle is not a limit point of the set $\left\{\varrho\left(\mathbf{A}_{N}\right)\right\}_{N=1}^{\infty}$ where $\varrho\left(\mathbf{A}_{N}\right)$ is the spectral radius of $\mathbf{A}_{N}$.

Under Assumption A1, no country is locally or globally dominant. No local dominance implies diversified foreign trade in each country in the sense of Definition

\footnotetext{
${ }^{8} h_{i} \equiv\left|\phi_{\pi i} \psi_{i i}+1-\phi_{\pi i}+\phi_{x i}+(1+\varphi) \lambda_{i} \phi_{\pi^{d} i}\right|$, and $m_{i} \equiv\left|\phi_{\pi i}\left(1-\psi_{i i}\right)\right|$
} 
1. Assumptions A1-A3 imply that the steady-state export shares $\chi_{N i j}$, defined as the share of exports from country $i$ to country $j$ on the GDP of country $i$, satisfy

$$
\chi_{N i j}=\frac{\psi_{N j i} \mathcal{P}_{j}}{\sum_{k=1}^{N} \psi_{N k i} \mathcal{P}_{k}}=\left\{\begin{array}{cc}
O(1) & i=j \\
O\left(N^{-1}\right) & i \neq j
\end{array},\right.
$$

which are the same order of magnitudes conditions as for the corresponding coefficients of import share matrix $\boldsymbol{\Psi}_{N}$. Assumption 4 ensures some regularity conditions on the sequence of import share matrices $\left\{\boldsymbol{\Psi}_{N}\right\}$ which are needed for $\lim _{N \rightarrow \infty} \chi_{N i i}$ to exist. Condition ( $i$ ) of Assumption A5 delivers determinacy of equilibrium for any $N$ sufficiently large. Assumption A5-(ii) is a weak condition that simplifies the analysis as it allows us to use some properties of strictly diagonally dominant matrices ${ }^{9}$. Assumption A5-(iii) establishes that $\varrho\left(\mathbf{A}_{N}\right)$ does not converge to one as $N \rightarrow \infty$, that is the set of eigenvalues of $\mathbf{A}_{N}$ is bounded away from unit circle.

TheOrem 2. Let Assumptions A1-A5 hold and suppose $\operatorname{rank}\left(\boldsymbol{\Psi}_{N}\right)=N-1$. Then as $N \rightarrow \infty$, the equilibrium solution for inflation and output gap given by (2.12) satisfies

$$
\left(\pi_{N i t}^{d}, x_{N i t}\right)^{\prime}-\mathbf{z}_{i t} \stackrel{q . m .}{\rightarrow} 0
$$

whereby $\mathbf{z}_{i t}$ is a $2 \times 1$ dimensional vector that solves the following system of countryspecific independent equations.

$$
\begin{aligned}
& \text { for any } i=1,2, \ldots, \text { where } \underset{2 \times 1}{\mathbf{z}_{i t}}=\left(\begin{array}{c}
\pi_{i t}^{d} \\
x_{i t}
\end{array}\right), \underset{2 \times 2}{\mathbf{G}_{0 i}}=\left(\begin{array}{cc}
1 & -(1+\varphi) \lambda_{i} \\
\phi_{\pi^{d} i} & 1-\phi_{\pi i}\left(1-k_{i}\right)+\phi_{x i}
\end{array}\right), \\
& \underset{2 \times 2}{\mathbf{G}_{1 i}}=\left(\begin{array}{cc}
\beta & 0 \\
1-\phi_{\pi i} & 1-\phi_{\pi i}\left(1-k_{i}\right)
\end{array}\right), \\
& \mathbf{G}_{2 i}=\left(\begin{array}{cc}
0 & 0 \\
-1 & \left(\rho_{a i}-1\right)\left(1-\phi_{\pi i}\left(1-k_{i}\right)\right)
\end{array}\right), \boldsymbol{\varepsilon}_{i t}=\left(\begin{array}{c}
\varepsilon_{r i t} \\
\varepsilon_{a i t}
\end{array}\right) .
\end{aligned}
$$

Proof is relegated to the Appendix.

Note that, although the structural form of the model involves parameters characterizing the openness of the economy, the reduced form of equation (3.3) is basically indistinguishable from the reduced form solution of a closed economy New Keynesian model, and is therefore only subject to domestic shocks.

That is, Theorem 2 states that as the number of countries is becoming large, the equilibrium of the multi-country model is in general arbitrarily well approximated by a solution to the independent country-specific systems of linear rational expectation equations that feature only domestic variables and domestic shocks. In other words, Theorem 2 establishes that for large $N$, dependence of an economy on the idiosyncratic shocks in the rest of the economies in the world is arbitrarily small.

Basic intuition is similar to the finance literature on portfolio diversification in a market with many assets (Chamberlain, 1983, and Chamberlain and Rothschild, 1983). Well diversified portfolio contains only factor variance, idiosyncratic risk is aggregated away. In the $\mathrm{N}$-country DSGE model, an impact of idiosyncratic shocks of foreign trade partners is aggregated away by diversifying exports and imports. In such a case the SOEF seems to be suitable. Note, however, that in our set

\footnotetext{
${ }^{9}$ This condition might well be redundant as it seems to be a necessary condition for A5(i) to hold. Note that the exact analytical expression for sufficient and necessary conditions for determinate equilibrium in general $N$ country model does not seem to exist.
} 
up, the rise in openness does not increase cross sectional dependence since trade diversification eliminates the international effects of idiosyncratic shocks.

Similar analogy can be also drawn with the econometric literature on large heterogenous panels with (multi)factor(s) error structure (Pesaran, 2006, Chudik and Pesaran, 2010). Here, as the number of cross section units tends to infinity, cross-sectional averages of dependent variables and regressors are used to wipe out the impact of underlying common factor(s). The degree of dependence of idiosyncratic shocks in our model was restricted by bounded eigenvalue of their covariance matrix, and therefore there is no systemic risk. If the cross section dependence of shocks were strong (in the sense defined in Chudik, Pesaran, and Tosetti, 2010), for example driven by a strong unobserved common factor(s), then the individual economies would depend only on the domestic shocks and the common factor(s). The cross section averages, or in the case of SOEF framework the representative foreign economy constructed as a cross section average of foreign economies, would be sufficient for capturing the effects of unobserved common factors. In what follows, we examine the features of the equilibrium when foreign trade flows are not diversified.

3.2. Locally Dominant Economies. The assumption that the world is populated by many small open economies which are similar in size and have diversified trade structure is not in line with the stylized facts. Smaller countries in terms of nominal GDP that are close to a large neighbor, such as Canada-US, tend to have a substantial proportion of the foreign trade with a large trade partner. The assumption of no local dominance is therefore likely to be a poor asymptotic approximation for the world economy. Assumption A1 is relaxed further and a finite number of economies are allowed to be locally dominant in the following Assumption A1.a.

Assumption A1.a (Foreign trade flows - case of locally dominant economies)

$$
\psi_{N i j}=\left\{\begin{array}{cl}
O\left(N^{-1}\right) & i \neq j \wedge(i, j) \notin \mathcal{Z} \\
O(1) \text { but not } o(1) & i=j \vee(i, j) \in \mathcal{Z}
\end{array}\right.
$$

where set $\mathcal{Z}$ represents fixed number of country pairs $(i, j)$ for which $\lim _{N \rightarrow \infty} \psi_{N i j}=$ $k_{i j} \neq 0$, i.e. no country is locally dominant with the exception of countries in $\mathcal{Z}$. For future reference, let $\mathbf{Z}_{\Psi}$ denote the smallest upper left submatrix of the infinitedimensional matrix $\boldsymbol{\Psi}_{\infty}$ that contains all country-pairs in $\mathcal{Z}$. Assumptions A1.a, A2, and A3 again imply that order of magnitudes of column sums of the export share matrices are equal to that of import share matrices.

Theorem 3. Assume A1.a, A2-A5 hold and $\operatorname{rank}\left(\boldsymbol{\Psi}_{N}\right)=N-1$. Then as $N \rightarrow \infty$, the unique stable equilibrium solution $\left(\pi_{N i t}^{d}, x_{N i t}\right)^{\prime} \stackrel{q . m .}{\rightarrow} \mathbf{z}_{i t}$ where $\mathbf{z}_{i t}=$ $\left(\pi_{i t}^{d}, x_{i t}\right)^{\prime}$ depends on domestic shocks only if country $i$ has diversified imports. If there exist country $j$, which is locally dominant for country $i$ then $\mathbf{z}_{i t}$ depend in general on the idiosyncratic shocks of countries $k \in \mathcal{M}_{i} \cup\{i\}$ where

$$
\mathcal{M}_{i} \equiv\left\{k \in \mathbb{N} ; \exists \text { directed path } \gamma_{i k} \text { connecting nodes } T_{i} \text { and } T_{k} \text { of graph } \Gamma\left(\mathbf{Z}_{\Psi}\right)\right\} \text {. }
$$

Proof is relegated to Appendix. For a definition of directed path $\gamma_{i k}$ and directed graph $\Gamma\left(\mathbf{Z}_{\Psi}\right)$ refer also to Appendix.

Theorem 3 implies that even if a number of countries are not locally dominant, small open economy framework is applicable for economies that diversify their exports and imports. Note, however, that in gravity models of trade, which enjoy a large empirical success in the literature, bilateral trade between economies is mainly a function of economic size and a measure of distance between countries. Therefore, 
it is more likely that trade weights in larger economies satisfy granularity conditions, as opposed to small economies, which often have a dominant neighbor. As a result, the small open economy framework is likely to be more suitable, somewhat paradoxically, for larger economies.

Note that if there exist a country pair $(i, j)$ for which $\psi_{N} i j \neq o(1)$ and $\psi_{N}, j i \neq$ $o(1)$, than these two countries need to be considered endogenously. More generally, Theorem 3 establishes that when country $i$ lies on the circle in the directed graph $\Gamma\left(\mathbf{Z}_{\Psi}\right)$, then all countries on this circle need to be considered endogenously. As a consequence of several simplifying assumptions imposed, matrix $\mathbf{A}_{N}=\mathbf{A}_{0, N}^{-1} \mathbf{A}_{1, N}$ in $N$-country solution (2.12) does not contain steady-state export share matrix (denoted below as $\Upsilon_{N}=\left[\chi_{N} i j\right]$ ). This is no longer true in a richer models, therefore the set $\mathcal{M}_{i}$ in Theorem 3 in general is a union of following sets $\mathcal{T}_{i} \cup\{i\}$ where

$\mathcal{T}_{i} \equiv\left\{1 \leq k \leq N ; \exists\right.$ directed path $\gamma_{i k}$ connecting nodes $T_{i}$ and $T_{k}$ of graph $\left.\Gamma\left(\mathbf{Z}_{\Upsilon}+\Psi\right)\right\}$, and $\mathbf{Z}_{\boldsymbol{\Upsilon}+\boldsymbol{\Psi}}$ denote the smallest upper left submatrix of the infinite-dimensional matrix $\boldsymbol{\Upsilon}_{\infty}+\Psi_{\infty}$ that contains all country-pairs $(i, j)$ such that either $(j, i) \in \mathcal{Z}$ or $(i, j) \in \mathcal{Z}$.

The intuition behind this result is as follows. Imagine country $i_{1}$ undertakes the majority of foreign trade with country $i_{2}$, while country $i_{2}$ has a large amount of foreign trade with countries $i_{1}$ and $i_{3}$. Then, despite no direct trade connections between countries $i_{1}$ and $i_{3}$, all three economies $\left(i_{1}, i_{2}, i_{3}\right)$ need to be considered endogenously. Since all agents are forward-looking, shocks to country $i_{3}$ can have instantaneous impact on country $i_{1}$.

Due to the limitations posed by the size of typical macroeconomic data sets, clear practical implication, which can be derived from Theorem 3, are mainly for small-large neighbor pairs, such as Switzerland-euro area or Canada-United States etc., or for countries that have important trade connection with any of its small neighbors (e.g. Slovakia and Czech Republic). For small economies that are dominated by a neighbor, the analysis suggests not to aggregate the rest of the world economies by ad-hoc trade-weights as it is commonly done, but to include variables from a dominant neighbor separately along the weighted averages of the remaining trade partners. This way, data are allowed to decide on the degree of the impact on the small domestic economy from a dominant neighbor compared to the impact form the rest of the world. The restrictions posed by the small open economy framework in the literature, that is aggregating all foreign variables into one fictional representative economy using trade weights, lead to a misspecification in the presence of locally dominant economies.

3.3. Globally Dominant Economies. According to International Monetary Fund's (IMF) International Financial Statistics (IFS) data, a few major industrialized economies account for a substantial fraction of the world's output. The USA alone accounts for about a $30 \%$ of the world's nominal GDP ${ }^{10}$. Furthermore, it is evident that certain economies account for a large share of exports and import in the world trade matrix. Hence Assumptions A1 and A3 are generalized further and we introduce the concept of a globally dominant economy(ies). This concept implies that $K$ countries represented in the set $\mathcal{K}=\left\{j_{1}, \ldots, j_{K}\right\}$ are allowed to be globally dominant, which means that countries in $\mathcal{K}$ represent a non-negligible fraction of world's GDP and $\sum_{i=1}^{N} \psi_{N} i j$ increases at the rate $N$ for $j \in \mathcal{K}$. In other words,

\footnotetext{
${ }^{10}$ Based on nominal exchange rates. The shares are computed using average GDP in the 20042006 period. The second largest economy is the euro area, which represents about 20 percent of aggregate GDP. See also Figure 1.
} 
countries in $\mathcal{K}$ are locally dominant for a sufficiently large number of economies in the world.

Assumption A1.b (Foreign trade flows - globally dominant economies)

$$
\psi_{N} i j=\left\{\begin{array}{cc}
O(1) & i=j \vee j \in \mathcal{K} \\
O\left(N^{-1}\right) & i \neq j, j \notin \mathcal{K}
\end{array} .\right.
$$

Furthermore, $\lim _{N \rightarrow \infty} \psi_{N i j}=k_{i j} \neq 0$, for $j \in \mathcal{K}$ with the exception of a finite number of country-pairs $(i, j), i \in \mathbb{N}, j \in \mathcal{K}$, for which $\lim _{N \rightarrow \infty} \psi_{N} i j=0$.

Assumption A3.a (Population)

$$
\begin{gathered}
\quad \frac{\mathcal{P}_{i}}{\mathcal{P}_{j}}=O(1) \text { for } i, j \in \mathbb{N} \backslash \mathcal{K} \text { and for } i, j \in \mathcal{K}, \\
\frac{\mathcal{P}_{i}}{\mathcal{P}_{j}}=O(N), \frac{\mathcal{P}_{j}}{\mathcal{P}_{i}}=O\left(N^{-1}\right) \text { for } i \in \mathcal{K} \text { and } j \in \mathbb{N} \backslash \mathcal{K} .
\end{gathered}
$$

Assumption A1.b states that no country is locally dominant with the exception of countries in $\mathcal{K}$. Furthermore, countries in $\mathcal{K}$ are locally dominant for almost all countries in the world economy. Local dominance of countries in $\mathcal{K}$ for almost all (i.e. with the exception of a finite number of) countries is a simplifying assumption that delivers easily tractable results. Assumption A1.b and A3.a could be slightly generalized in the light of the previous analysis, namely local dominance could be allowed by assuming $\lim _{N \rightarrow \infty} \psi_{N} i j$ is nonzero for fixed number of country-pairs $(i, j \notin \mathcal{K})$. This generalizations would not bring any new additional insights and it is therefore not pursued further here. Assumptions on import shares and population, A1.b, A2, A3.a, imply that

$$
\sum_{i=1}^{N} \chi_{N} i j=\left\{\begin{array}{cc}
O(N) & j \in \mathcal{K} \\
O(1) & j \notin \mathcal{K}
\end{array} \quad \text { and } \chi_{N i j}=\frac{\psi_{N}{ }_{j i} \mathcal{P}_{j}}{\sum_{k=1}^{N} \psi_{N}{ }_{k i} \mathcal{P}_{k}} \neq o(1) \text { for } j \in \mathcal{K}\right.
$$

The column sums of the export share matrix corresponding to globally dominant economies diverge to infinity at the rate $N$, similarly to the import share matrix. The column sums for the small economies remain bounded.

Theorem 4 (Globally dominant economies). Assume A1.b,A2,A3.a, and A4A5 hold, and suppose $\operatorname{rank}\left(\boldsymbol{\Psi}_{N}\right)=N-1$. Then as $N \rightarrow \infty$, countries in $\mathcal{K}$ become common factors in the world economy. Equilibrium solution for countries in $\mathcal{K}$ depends on idiosyncratic shocks in all countries in $\mathcal{K}$, i.e. countries in $\mathcal{K}$ need to be considered endogenously. Equilibrium solution for country $i \notin \mathcal{K}$ depends only on idiosyncratic shocks in country $i$ and idiosyncratic shocks in countries in $\mathcal{K}$.

Proof is again relegated to Appendix.

An important implication of Theorem 4 is that globally dominant countries become common factors for all countries in the world. Furthermore, globally dominant countries need to be considered endogenously since the equilibrium solution for country $i \in \mathcal{K}$ depend on the idiosyncratic shocks of all countries in $\mathcal{K}$. This is true even if country $i \in \mathcal{K}$ diversifies its exports and imports. ${ }^{11}$ Idiosyncratic shock in any country $j \notin \mathcal{K}$ is irrelevant for countries in $\mathcal{K}$, as these shocks are aggregated away.

With regards to small economies, as long as their exports and imports to and from their foreign trade partners that do not belong to $\mathcal{K}$ are diversified, equilibrium is affected only by domestic shocks and by shocks from globally dominant economies. Impact of a globally dominant economy $i \in \mathcal{K}$ on a small economy

\footnotetext{
${ }^{11}$ Country $i \in \mathcal{K}$ cannot become independent from the idiosyncratic shocks of any other globally dominant country $j \in \mathcal{K}$, solely due to its size, even though there could be no direct trade connection between $i$ and $j$.
} 
$j \notin \mathcal{K}$ cannot be avoided in general, even if there is no direct trade connection between the two countries. If a small economy $j \notin \mathcal{K}$ is locally dominant for economy $i \notin \mathcal{K}$, and vice versa, then these two small economies should be considered endogenously, besides common factors (countries in $\mathcal{K}$ ). Theorem 3 provides, therefore, a more general theoretical characterization of the countries that should be considered endogenously.

The United States is obviously the top candidate for the globally dominant economy. Assuming it is the only globally dominant economy, the immediate practical implication of Theorem 4 is to include US variables separately along with the remaining foreign variables (trade-weighted averages plus possibly other locally dominant economies) in the model for non-US economies. This is regardless of the amount of the direct foreign trade of a particular domestic economy under consideration with the US.

In the next section, we will apply the results derived above to assess the extent of global macroeconomic interdependence using our calibrated model.

\section{Assessing Macroeconomic Interdependence}

This section provides some stylized facts on the size, the trade openness and the degree of macroeconomic interdependence using data for 153 countries. We start by inspecting the size of the economies and their foreign trade share. Figure 1 presents data on the 12 largest economies according to the nominal GDP. 16 Eurozone countries that joined Euro (as of 2010) are treated as a single economy, referred to as the euro area. ${ }^{12}$ The US is the largest economy (followed by the euro area, Japan, China and the UK), but it is less open than the euro area, which is the most open from this group in terms of aggregate foreign trade. Figure 2 presents the column sums of the export and import share matrices as well as the foreign trade share matrix. ${ }^{13}$ Note that we construct the proxies for the model parameters $\psi_{i j}$ (the share of country $j$ imports in consumption of country $i$ ) and $\chi_{i j}$ (the share of exports to country $j$ in GDP of country $i$ ) using foreign trade data from the IMF Direction of Trade Statistics (DOTS) database. The data on GDP is taken from IMF IFS database. Column sums in all these matrices are largest for the Euro area, followed by the US.

We also provide some statistics on the relationship between openness and trade diversification. In theory, an increase in openness could lead to both, an increase or reduction in trade diversification. In fact, globalization and reduction in trade costs are likely to reduce the relevance of locally dominant neighbor and increase the importance of economies in the periphery. This means in our framework that an increase in openness could lead in fact to a reduction of macroeconomic interdependence. In Figure 3, we plot a measure of openness by dividing the aggregate foreign trade flows (exports + imports) by the GDP during 2004-06. The most open economy according to our measure are smaller countries, such as Malaysia, while the United States is relatively closed.

In Figure 4, we plot the degree of trade diversification calculated by the squared Euclidian norm of the trade weights. Canada and Mexico, which have also a high

\footnotetext{
${ }^{12}$ Austria, Belgium, Cyprus, France, Germany, Italy, Luxembourg, Malta, Netherlands, Finland, Greece, Ireland, Portugal, Slovakia, Slovenia and Spain.

${ }^{13}$ For details, please refer to Appendix. We also construct the foreign trade share matrix, with its element $(i, j)$ being $\left(I M_{i j t}+E X_{i j t}\right) /\left(\sum_{j=1}^{N} I M_{i j t}+\sum_{j=1}^{N} E X_{i j t}\right)$, that is the share of nominal exports and imports to/from a particular country $j$ on the total foreign trade in country $i$. This measure is directly observable and therefore not subject to data issues in construction of matrices $\Upsilon, \Psi$.
} 
degree of openness, appear to have less diversified trade structure. This indicates that both economies are likely to be subject to shocks of a locally dominant neighbor, namely the United States. Euro area, on the other hand, which has higher degree of openness compared to the US, is also more diversified then the US according to our measure. In fact, Figure 5 shows that there is no clear-cut relationship between the two variables. The relationship between the two variables is ambiguous, i.e. open economies are not necessarily subject to a more diversified trade structure.

In order to investigate the degree of macroeconomic interdependence across economies, we also construct probability impulse-response (PIR) functions for the 153 economies in our sample. Probability impulse response functions are well suited for identifying the importance of foreign shocks in an environment characterized by uncertainty. We calculate PIR functions according the following procedure. First, for simplicity we assume that all standard deviations of the exogenous shocks are equal $^{14}$. Second, we calculate the impact of a positive ( 2 standard deviations) monetary policy shock $\varepsilon_{r i t}$ on the remaining countries in the sample, while at the same time we draw random values for the remaining structural shocks from their corresponding probability distribution. Third, we calculate the probability of the variable $\pi_{j, t+h}^{d}$ being below its steady-state value, where $h \geq 0$ is the time horizon. Clearly, if the probability in country $j$ is at 50 percent, i.e. the probability impulse response of inflation is equally likely to be below or above its steady-state value, than a monetary policy shock in country $i$ has no expected impact on $\pi_{j, t+h}^{d}$. More formally, we define the probability impulse-response function as being:

$$
P I R_{r, \pi^{d}}(i, j, h)=P\left(\pi_{j h}^{d}<E\left(\pi_{j h}^{d}\right) \mid \varepsilon_{r i, 0}=2 \sigma_{\varepsilon_{r i}}^{2}, \varepsilon_{-1}=\mathbf{0}, \boldsymbol{\varepsilon}_{-2}=\mathbf{0}, \ldots\right) .
$$

The probability impulse response functions are computed numerically using the following formula:

$$
\operatorname{PIR}_{r, \pi^{d}}(i, j, h) \approx \frac{1}{R} \sum_{s=1}^{R} I\left(\pi_{j h}^{d, s}\right),
$$

where $I\left(\pi_{j h}^{d, s}\right)$ is indicator function giving value 1 if $\pi_{j h}^{d, s}<E\left(\pi_{j h}^{d, s}\right)$ and value zero otherwise. $\pi_{j h}^{d, s}$ is value of variable $\pi_{j h}^{d}$ in the $s$-th replication of the experiment, $s \in\{1,2, \ldots, R\}$.

With regards to the calibration of the model, the multicountry model is simulated with the import share matrix $\boldsymbol{\Psi}$ constructed from 2004-2006 data for 153 countries representing virtually all output in the world (99.1\%). We estimate the $\operatorname{AR}(1)$ coefficient in the exogenous productivity process $\rho_{a}=0.22$ using annual data on real GDP per capita for the period 1965-2005. We assume that this parameter is homogenous across the sample. Monetary policy functions (2.5) are also postulated to be homogenous with $\phi_{\pi^{d} i}=3, \phi_{\pi i}=1, \phi_{x i}=0.3$ for all $i$. Time profiles of the shock and the magnitudes of the impact are sensitive to the value of the parameters, but the relative ranking of the contemporaneous impact of a shock across countries is so to a lesser extent. Thus we will focus on the contemporaneous impact, $h=0$ only. Table 2 in Appendix summarizes the remaining parameters of the model.

Results for the two largest economies, the United States and the euro area, and two small economies, Switzerland and Canada, are presented in Figure 6. Two types of charts are constructed for each economy. Charts on the left present the value of the probability impulse-response function of domestic PPI at horizon $h=0$ following a shock to foreign monetary policy. Only the top 10 countries with the

\footnotetext{
${ }^{14}$ Note that only the relative size, but not the absolute magnitude of the standard deviations is important for the construction of the probability impulse response function.
} 
largest impact on the home economy are presented. The charts on the right show the value of the probability impulse-response function of foreign PPI at horizon $h=0$ following a domestic monetary policy shock. Again, only the top 10 of most affected economies are presented. Thus, the charts on the left hand side identify which foreign monetary policy shocks have the largest contemporaneous impact on domestic PPI, while the charts on the right hand side define the set of foreign economies that are mostly affected by domestic monetary policy shocks.

The results presented in the upper two sets of charts show that monetary policy shocks in the euro area and the United States have a substantial impact on a number of foreign economies. While US monetary policy shocks have a strong impact particularly on Northern and Central American economies, euro area monetary policy shocks are affecting mainly Central and Eastern European economies. Foreign monetary policy shocks, however, have only minor impact on domestic PPI in the euro area and the United States. Interestingly, however, US and euro area shocks have a relatively mild impact on each other. Thus the casual investigation of Figure 6 suggests that while both economies are locally dominant for a number of foreign countries, the overall affect on each other via the presented trade channel might be small. Recall, however, that capital markets are treated in the theoretical model symmetrically (as asset markets are assumed to be complete), thus the role of United States could be underestimated in Figure 6 .

The results for Canada and Switzerland (bottom two sets of charts in Figure 6) document the presence of a locally dominant neighbors: in the case of Canada, the United States, while in the case of Switzerland, the euro area. The fact that Canada and Switzerland are rather small is documented in the charts on the right hand side. Monetary policy shock to either of these two economies have a negligible impact on the PPI inflation in the any of the remaining economies in the world.

\section{Concluding Remarks}

How does openness and size of the economy affect macroeconomic interdependence? Consensus in the profession is that an increase in openness results in an increased dependence of home economy on foreign shocks. Such prediction is supported by the existing small open economy models in the literature. In this paper, we challenged this traditional view by using a multicountry general equilibrium as a laboratory. First, we demonstrated the conditions under which a well-defined equilibrium of an $N$-country model exists. Second, by using large $N$ asymptotics, we showed that, in contrast with the established consensus, an increase in openness might well be associated with decreased dependence on foreign shocks. Our analysis suggests that it is not the openness per se that is important for macroeconomic interdependence. A key to macroeconomic independence from the idiosyncratic shocks to other economies in a globalized world is the ability of a country to diversify its exports and imports. Furthermore, there does not seem to be a clear link between the change in openness and the diversification of trade flows.

Analysis of macroeconomic interdependence within the context of a multicountry model allows us to make additional interesting observation. We demonstrated that the criteria of economic smallness and/or openness cannot be used to justify the so-called Small Open Economy Modelling Framework (SOEF), which is perhaps the most commonly used approach in the applied open economy literature to overcome the dimensionality problem. In one of our examples, where the world consisted of one globally dominant economy and many small economies, the so-called 'SOEF data shrinkage' would be justifiable for the dominant economy only, whereas it could lead to highly misleading conclusions for any of the small economies. As a 
result, our analysis, although being purely theoretical in nature, contains important implications for applied empirical work, as well.

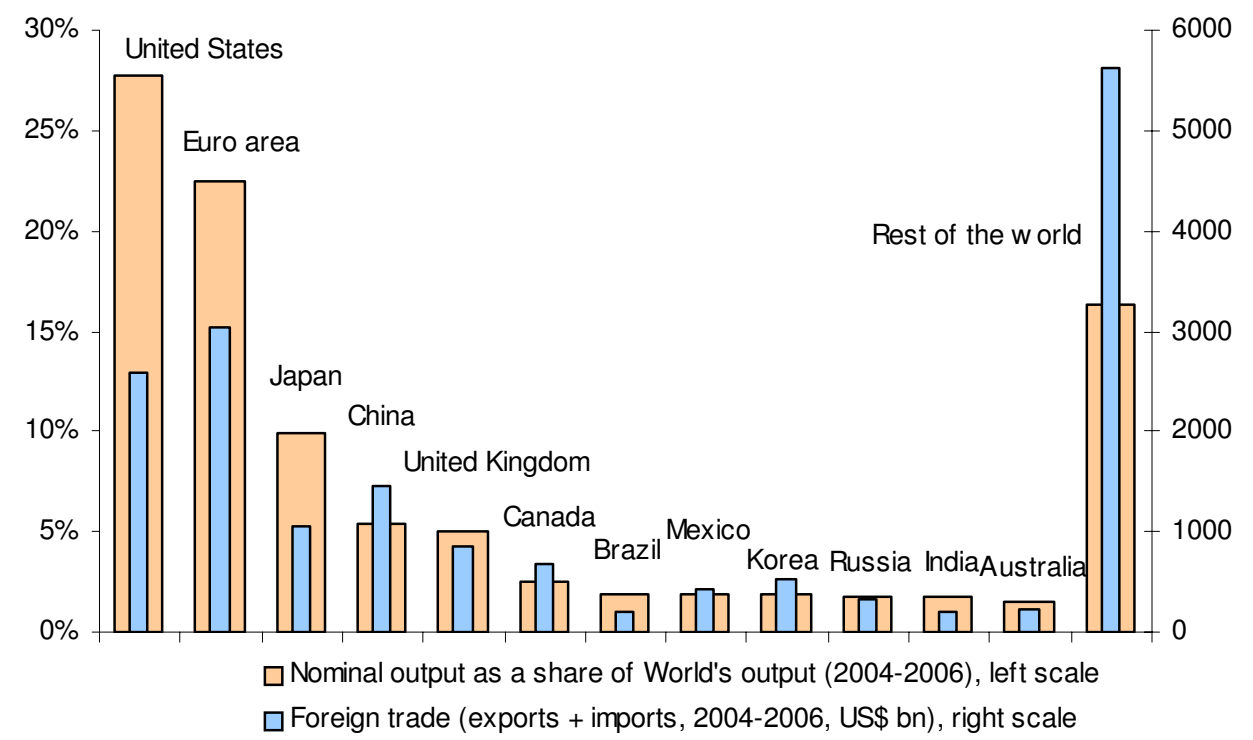

Figure 1: Nominal Output and Foreign Trade (Top 12 largest economies according to nominal GDP)

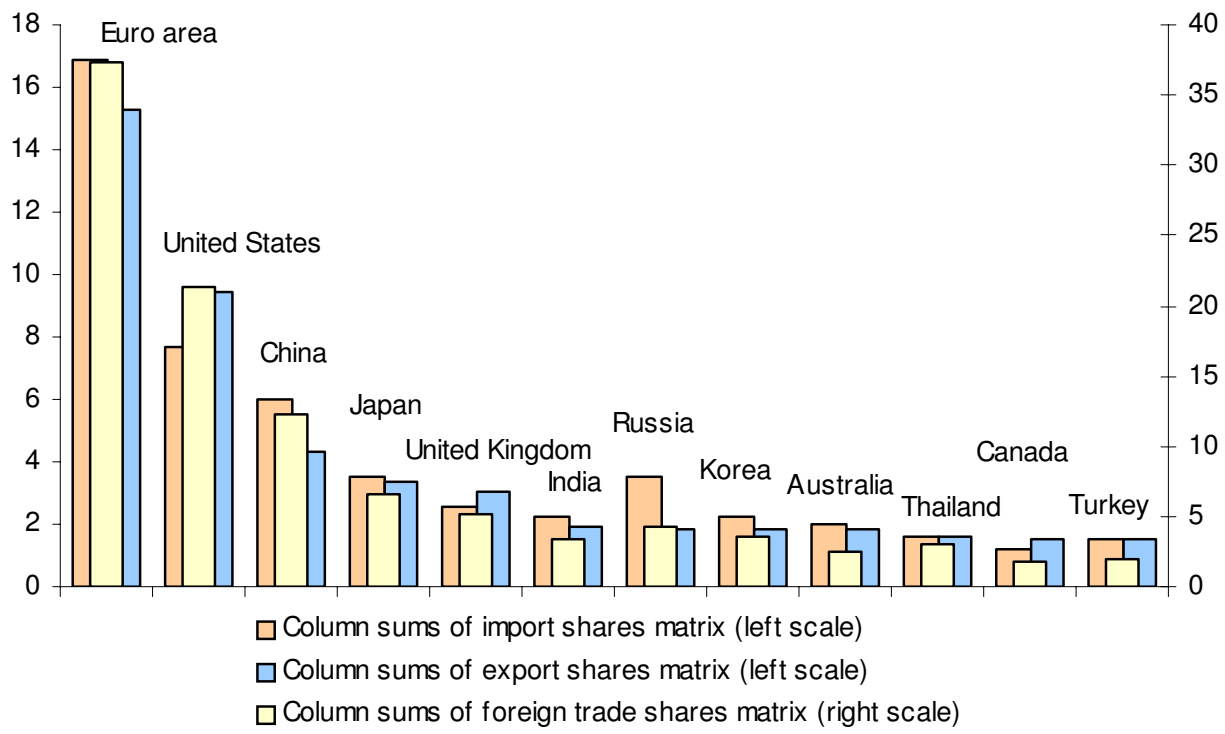

Figure 2: The Largest Column Sums of Export, Import and FT Matrices, 2001-2003 average. 


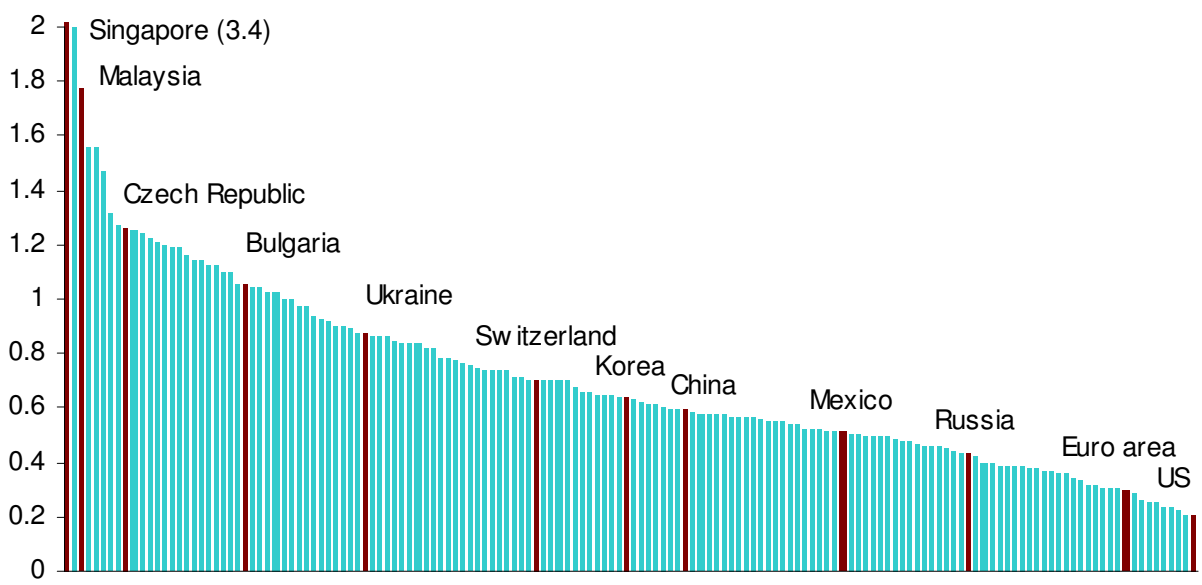

FIGURE 3: Economic openness (export + imports as a share of GDP, 2004 - 2006)

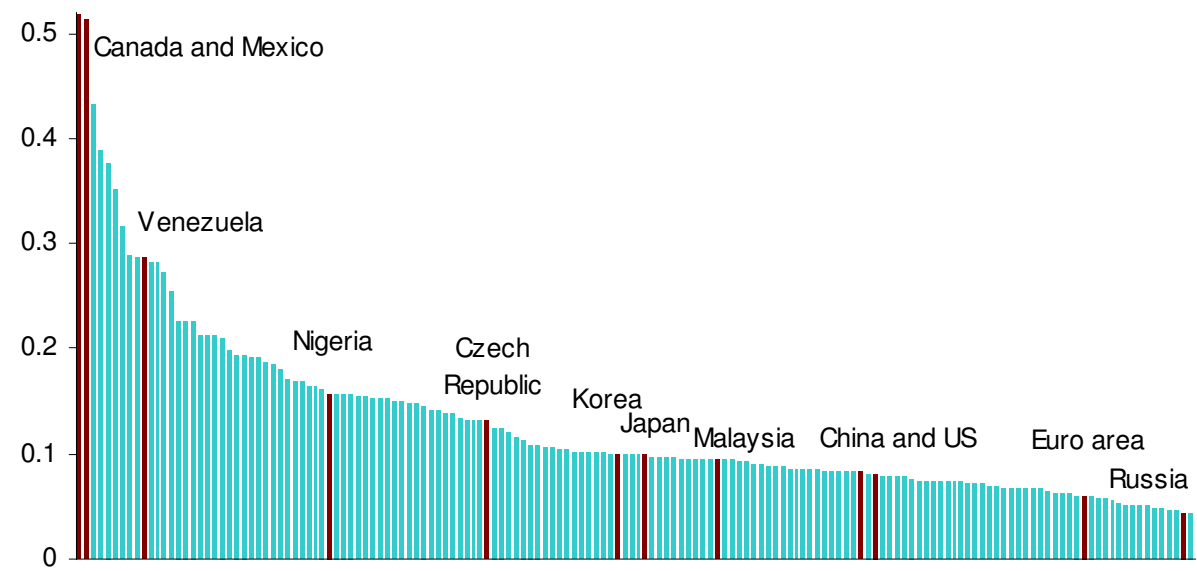

FIGURE 4: Squared euclidian norm of foreign trade weights vectors (2004-06)

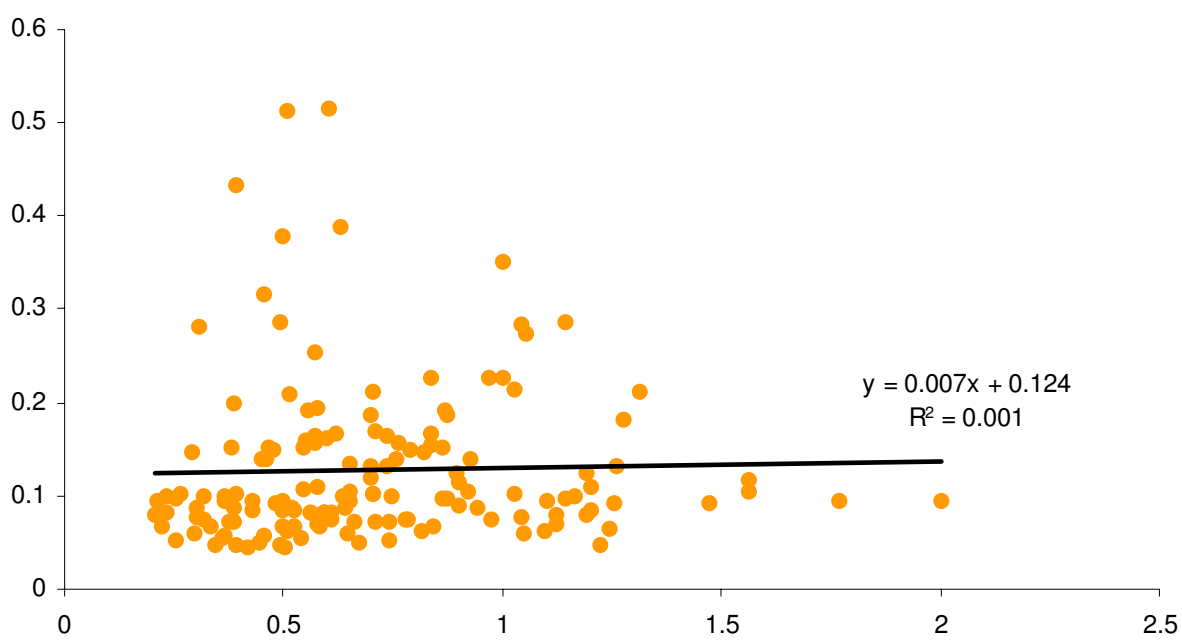

FiguRE 5: Squared euclidean norm of weights vectors (Y-axis) vs. openess (X-axis) 


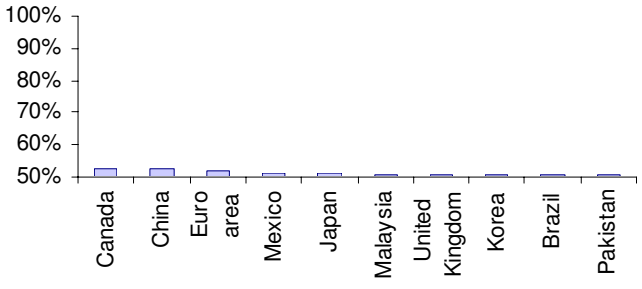

$\square$ Impact of a foreign monetary policy shock on domestic (United States) inflation (top 10 countries $w$ ith the largest impact)

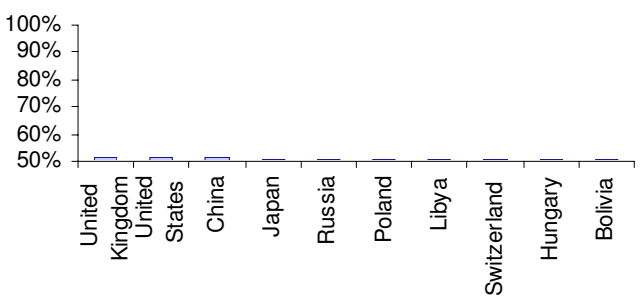

$\square$ Impact of a foreign monetary policy shock on domestic (EU) inflation (top 10 countries $w$ ith the largest impact)

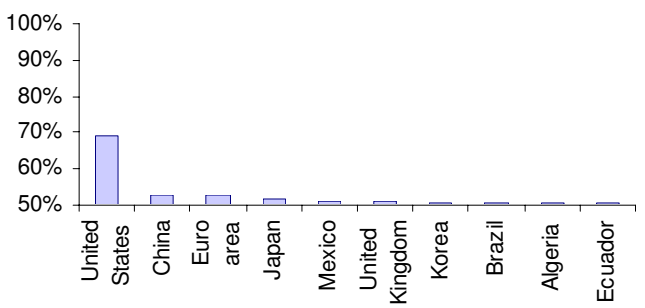

$\square$ Impact of a foreign monetary policy shock on domestic (Canada) inflation (top 10 countries $w$ ith the largest impact)

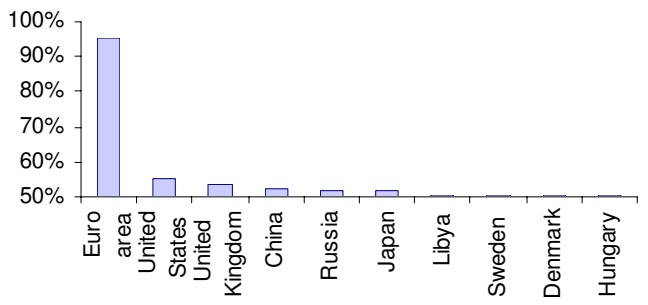

$\square$ Impact of a foreign monetary policy shock on domestic (Sw itzerland) inflation (top 10 countries $w$ ith the largest

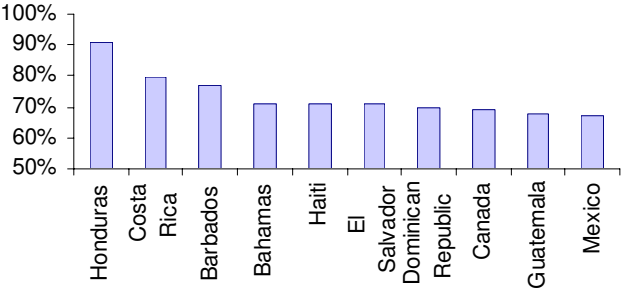

$\square$ Impact of a domestic (United States) monetary policy shock on foreign inflation (top 10 most affected countries)

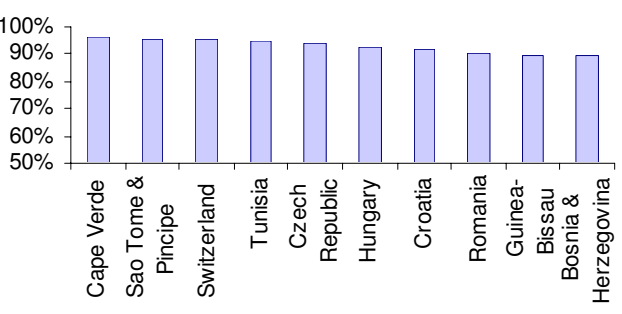

$\square$ Impact of a domestic (Euro area) monetary policy shock on foreign inflation (top 10 most affected countries)

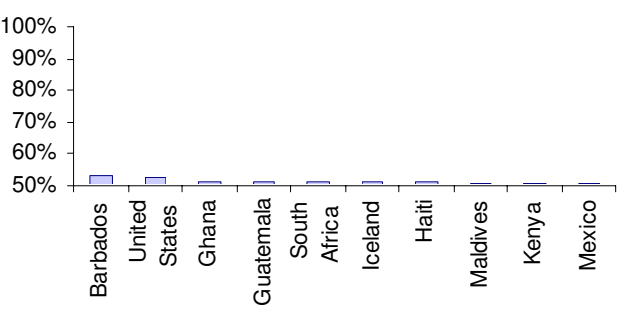

$\square$ Impact of a domestic (Canada) monetary policy shock on foreign inflation (top 10 most affected countries)

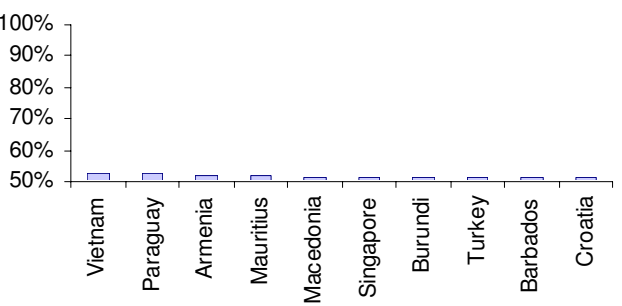

$\square$ Impact of a domestic (Sw itzerland) monetary policy shock on foreign inflation (top 10 most affected countries)

FiguRE 6: Probability impulse-response functions at the time of impact $(h=0)$ : Top 10 most affected countries. 
Appendix A. Detailed description of model and its solution

\section{A.1. Detailed Description of Model's Assumptions.}

A.1.1. Households. The world consists of $N$ countries indexed by $i \in\{1,2, \ldots, N\}$ where country 1 is chosen as a numeraire. Each country is populated by three types of agents: population $\mathcal{P}_{i}$ of households, by a unit mass of firms and a central bank. Each household has the following felicity function separable in labor and consumption and logarithmic in consumption for simplicity:

$$
u\left(C_{i t}, \stackrel{\circ}{L}_{i t}\right)=\ln C_{i t}-\frac{\stackrel{\circ}{L}_{i t}^{1+\varphi}}{1+\varphi},
$$

where $\stackrel{\circ}{L}_{i t}$ denotes common level of supplied labor across households in country $i$. It also represents per capita employment. Aggregate employment (total hours worked) in country $i$ is denoted by

$$
L_{i t}=\stackrel{\circ}{L}_{i t} \mathcal{P}_{i}
$$

$C_{i t}$ represents consumption index defined below. Tradable goods produced possibly all around the world enter $C_{i t}$. Cobb-Douglas preferences over tradeable goods from different countries are assumed for simplicity:

$$
C_{i t}=\frac{1}{\prod_{j=1}^{N} \psi_{i j}^{\psi_{i j}}} \prod_{j=1}^{N} C_{i j t}^{\psi_{i j}},
$$

where

$$
\psi_{i j} \geq 0, \sum_{j=1}^{N} \psi_{i j}=1,
$$

and $C_{i j t}$ is consumption index of goods imported from country $j$ and consumed by household in country $i$. Each economy is open and there is a home bias in consumption, that is, nominal consumption shares of tradeables $\psi_{i j}$ satisfy

$$
1>\psi_{i i}>\max _{j \neq i} \psi_{i j} \quad \forall i \in\{1,2, \ldots, N\} .
$$

It is also assumed that no group of countries is isolated from the rest of the world. Lemma 2 implies that this is the case if and only if

$$
\operatorname{rank}\left(\mathbf{I}_{N}-\underset{N \times N}{\mathbf{\Psi}}\right)=N-1,
$$

where $(i, j)$-th coefficient of import share matrix $\boldsymbol{\Psi}$ equals $\psi_{i j}$ and $\mathbf{I}_{N}$ is $N \times N$ identity matrix.

LemMa 2. World consists of $K \geq 1$ isolated groups of countries if and only if

$$
\operatorname{rank}\left(\mathbf{I}_{N}-\mathbf{\Psi}\right)=N-K
$$

where $\boldsymbol{\Psi}$ is any import share matrix defined by (A.3).

Proof is in Appendix B. ${ }^{15}$

There is a continuum of goods produced in each country. Goods made in country $j$ are located on an interval denoted by $I_{j} ; I_{j} \cap I_{i}=\emptyset$ for $i \neq j$. Dixit-Stiglitz preferences over goods produced in the same country are assumed,

$$
C_{i j t}=\left(\int_{I_{j}} \mathfrak{C}_{i t}^{\frac{\epsilon-1}{\epsilon}}(\ell) d \ell\right)^{\frac{\epsilon}{\epsilon-1}}
$$

$\mathfrak{C}_{i t}(\ell)$ denotes actual consumption of good $\ell$ (produced possibly abroad) by household in country $i$. Elasticity of substitution $\epsilon$ is for simplicity postulated to be the same across countries.

\footnotetext{
${ }^{15}$ Notice that matrix $\left(\Psi-\mathbf{I}_{N}\right)$ can never have full rank since the row-sum of any row of import share matrix $\boldsymbol{\Psi}$ equals one.
} 
Denote the corresponding price index (i.e. the minimum expenditure for one unit of the consumption index $C_{i t}$ ) by $P_{i t}$. With preferences (A.2) and (A.7), CPI is given by familiar formulae

$$
P_{i t}=\prod_{j=1}^{N} P_{i j t}^{\psi_{i j}}, P_{i j t}=\left(\int_{I_{j}} \mathfrak{P}_{i t}^{1-\epsilon}(\ell) d \ell\right)^{\frac{1}{1-\epsilon}},
$$

where $\mathfrak{P}_{i t}(\ell)$ denotes consumer price of $\operatorname{good} \ell$ in country $i$. All prices are expressed in local currency. International trade is costly. Iceberg shipping costs imply that any good $\ell \in I_{j}$ costs $\mathfrak{P}_{i t}(\ell)$ in country $i$, where

$$
\mathfrak{P}_{i t}(\ell)=E_{i j t} \Theta_{i j} \mathfrak{P}_{t}^{d}(\ell) \quad \text { for } \ell \in I_{j},
$$

and $\mathfrak{P}_{t}^{d}(\ell)$ is the producer price of good $\ell$ expressed in domestic currency. Superscript $d$ is used to denote producer prices and/or indices. $\left(\Theta_{i j}-1\right)$ represents tariff equivalent of the iceberg shipping $\operatorname{costs} \tau_{i j}$,

$$
\Theta_{i j}=\frac{1}{1-\tau_{i j}} .
$$

It is assumed that shipping cost obey strict triangle inequality

$$
\tau_{i j}<\tau_{k j}+\tau_{i k} \quad \forall i, j \text { and } \forall k \neq j, i,
$$

so that it is not profitable to ship goods from country $j$ to country $i$ via importing to and exporting from the third country $k \neq j, i$. No distribution costs within a country implies $\tau_{i i}=0, \forall i \in\{1,2, \ldots, N\} . E_{i j t}$ is exchange rate between country $i$ and $j$. It is defined as the amount of currency $i$ per one unit of currency of country $j$. For convenience, country 1 is chosen as a numeraire country and exchange rate of country $i$ relative to the numeraire country 1 is denoted as $E_{i t} .{ }^{16}$ In this way, bilateral exchange rate $E_{i j t}$ can be written equivalently as

$$
E_{i j t}=\frac{E_{i t}}{E_{j t}} .
$$

It is useful to define for future reference also the Producer Price Index (PPI) of country $i$ as

$$
P_{i t}^{d} \equiv\left(\int_{I_{i}}\left[\mathfrak{P}_{t}^{d}(\ell)\right]^{1-\epsilon} d \ell\right)^{\frac{1}{1-\epsilon}} .
$$

Above preferences of households (A.2) and (A.7) imply well-known demand functions. With Cobb-Douglas preferences (A.2), households spend share $\psi_{i j}$ of their total spending on goods imported from country $j$,

$$
P_{i j t} C_{i j t}=\psi_{i j} P_{i t} C_{i t} .
$$

Dixit-Stiglitz preferences (A.7) imply familiar implicit demand functions for individual goods,

$$
\mathfrak{C}_{i t}(\ell)=\left(\frac{\mathfrak{P}_{i t}(\ell)}{P_{i j t}}\right)^{-\epsilon} C_{i j t} \text { for } \ell \in I_{j}
$$

Household in country $i$ maximizes lifetime utility

$$
\mathcal{U}_{i t}=\max _{\left\{C_{i, t+k}, \stackrel{\stackrel{L}{L}_{i, t+k}}{\mathcal{S}_{i, t+k}}\right\}_{k=0}^{\infty}} \mathrm{E}_{t} \sum_{k=0}^{\infty} \beta^{k} u\left(C_{i, t+k}, \stackrel{\circ}{L}_{i, t+k}\right),
$$

where $\mathcal{S}_{i t}$ is the set of asset holdings of a household in country $i$. Households maximize utility function subject to the sequence of budget constraints, which can be written following Gali and Monacelli (2005) as

$$
P_{i t} C_{i t}+\mathrm{E}_{t}\left\{\mathfrak{M}_{i t, t+1} D_{i, t+1}\right\}=D_{i t}+W_{i t} \stackrel{\circ}{L}_{i t},
$$

\footnotetext{
${ }^{16}$ Selection of a numeraire is irrelevant.
} 
where $D_{i, t+1}$ represents nominal payoff in period $t+1$ of the portfolio held by a household in country $i$ at the end of period $t$ (including shares). It is assumed that both domestic and international asset markets are complete. $\mathfrak{M}_{i t, t+1}$ is relevant stochastic discount factor. With felicity function (A.1) the discount factor is given by

$$
\mathfrak{M}_{i t, t+1}=\beta \frac{C_{i t}}{C_{i, t+1}} \frac{P_{i t}}{P_{i, t+1}} .
$$

Taking directly logarithm of defined consumer price index (A.8) and substituting (A.9) yields relationship between consumer and producer price indices, which can be written in matrix form as

$$
\mathbf{p}_{t}-\mathbf{e}_{t}=\Psi\left(\mathbf{p}_{t}^{d}-\mathbf{e}_{t}\right)+\boldsymbol{\vartheta}_{1}
$$

where $\mathbf{p}_{t}=\left(p_{1 t}, \ldots, p_{N t}\right)^{\prime}, \mathbf{e}_{t}=\left(0, e_{2 t}, \ldots, e_{N t}\right)^{\prime}, \mathbf{p}_{t}^{d}=\left(p_{1 t}^{d}, \ldots, p_{N t}^{d}\right)^{\prime}$ and $i$-th element of vector $\boldsymbol{\vartheta}_{1}$ is $\vartheta_{1 i}=\sum_{j=1}^{N} \psi_{i j} \theta_{i j} .{ }^{17}$ Link between consumer and producer price indices is used in derivations below.

Households' Optimization Problem. First order conditions to household optimization problem with felicity function given by (A.1) imply standard intratemporal optimality condition in log-linear terms:

$$
\varphi i_{i t}+c_{i t}=w_{i t}-p_{i t} .
$$

It is also useful to write the first order condition for policy instrument - one period bond with gross return $R_{j t}$ denominated in the currency of country $j$. If this bond is bought by household in country $i$, then the return in the currency of country $i$ is $R_{j t}\left(E_{i j, t+1} / E_{i j t}\right)$.

$$
\mathrm{E}_{t}\left\{\mathfrak{M}_{i t, t+1}\left(R_{j t} \frac{E_{i j, t+1}}{E_{i j t}}\right)\right\}=1 .
$$

Log-linearization of first order condition (A.19) for household in country $i$ and policy instrument $R_{i t}$ yields standard intertemporal optimality condition ${ }^{18}$

$$
c_{i t}=\mathrm{E}_{t}\left\{c_{i, t+1}\right\}-\left(r_{i t}-\mathrm{E}_{t}\left\{\pi_{i, t+1}\right\}+\ln \beta\right),
$$

where $\pi_{i t} \equiv \Delta p_{i t}$ is consumer price inflation in country $i$, and $r_{i t}=\ln R_{i t}$. Subtracting log-linearized asset market arbitrage condition (A.19) for household in country $i$ and instruments $R_{j t}, R_{i t}$ yields standard uncovered interest parity condition

$$
\mathrm{E}_{t}\left\{\Delta e_{i j, t+1}\right\}=\mathrm{E}_{t}\left\{\Delta e_{i, t+1}-\Delta e_{j, t+1}\right\}=r_{i t}-r_{j t} .
$$

A.1.2. Firms. Linear production function is assumed for simplicity with the only input being labor

$$
\mathfrak{Y}_{t}(\ell)=A_{i t} L_{t}(\ell) \quad \text { for } \ell \in I_{i},
$$

where $\mathfrak{Y}_{t}(\ell)$ and $L_{t}(\ell)$ denote output and employment of firm $\ell$, respectively. Various exogenous processes for the productivity processes could be assumed. This appendix allows for productivity processes that are not necessarily cointegrated across countries. Let $f_{a t}$ denote common factor defined as

$$
\Delta f_{a t}=\zeta_{f_{a} t}
$$

where $\zeta_{f_{a} t}$ is independently and identically distributed $(i i d)$ with $\mathrm{E}\left(\zeta_{f_{a} t}\right)=0, \mathrm{E}\left(\zeta_{f_{a} t}^{2}\right)=$ $\sigma_{\zeta_{f a}}^{2}$. Let $\varepsilon_{a i t}$ denote idiosyncratic stationary weakly cross-sectionally dependent (CWD)

\footnotetext{
${ }^{17}$ Lower case letters denote logarithms, i.e. $p_{i t}=\ln \left(P_{i t}\right), p_{i t}^{d}=\ln \left(P_{i t}^{d}\right), e_{i t}=\ln \left(E_{i t}\right)$ and $\theta_{i j}=\ln \left(\Theta_{i j}\right)$.

${ }^{18}$ See for example Gali and Monacelli (2005). Adopted solution technique (log-linearization around a steady-state) is satisfactory only if the deviations from equilibrium are not large. A steady-state of the world economy is formally defined and analyzed in Chudik (2008) and briefly summarized in Appendix A.4. Steady-state solution for variables featuring in the first order condition (A.19) is $\Delta p_{i t}=0, \Delta e_{i t}=0, \Delta c_{i t}=0$ and $r_{i t}=-\ln \beta$.
} 
$\operatorname{AR}(1)$ process, ${ }^{19}$

$$
\varepsilon_{a i t}=\rho_{a i} \varepsilon_{a i, t-1}+\zeta_{\varepsilon_{a} i t}, \text { for } i=1,2, . ., N
$$

where $\left|\rho_{a i}\right|<\rho<1$, individual disturbances collected into vector $\boldsymbol{\zeta}_{\varepsilon_{a} t}=\left(\zeta_{\varepsilon_{a} 1 t}, \ldots, \zeta_{\varepsilon_{a} N t}\right)^{\prime}$ have zero mean, $\mathrm{E}\left(\boldsymbol{\zeta}_{\varepsilon_{a} t}\right)=\mathbf{0}$, and nonnegative definite covariance matrix $\mathrm{E}\left(\boldsymbol{\zeta}_{\varepsilon_{a} t} \boldsymbol{\zeta}_{\varepsilon_{a} t}^{\prime}\right)=$ $\boldsymbol{\Sigma}_{\varepsilon_{a}}$. Process $\boldsymbol{\zeta}_{\varepsilon_{a} t}$ is serially uncorrelated and CWD, particularly the spectral radius of covariance matrix, denoted by $\varrho\left(\boldsymbol{\Sigma}_{\varepsilon_{a}}\right)$, is bounded in $N$. Following technology process is assumed.

$$
\Delta a_{i t}=\gamma \Delta f_{a t}+(1-\gamma) \zeta_{a i t}+\Delta \varepsilon_{a i t}
$$

where $\boldsymbol{\zeta}_{a t}=\left(\zeta_{a 1 t}, \ldots, \zeta_{a N t}\right)^{\prime}$ is weakly dependent serially uncorrelated process with zero mean, $\mathrm{E}\left(\boldsymbol{\zeta}_{a t}\right)=\mathbf{0}$, and nonnegative definite covariance matrix $\mathrm{E}\left(\boldsymbol{\zeta}_{a t} \boldsymbol{\zeta}_{a t}^{\prime}\right)=\boldsymbol{\Sigma}_{a}, \varrho\left(\boldsymbol{\Sigma}_{a}\right)=$ $O(1)$. Pesaran and Tosetti (2010) show that all common spatial processes are CWD. Hence the definition of productivity process (A.24) allows for relatively rich pattern of cross-sectional dependence. If $\gamma=1$, then productivity processes are cointegrated across countries. Otherwise productivity across countries are not cointegrated, but there is a common nonstationary factor for $\gamma \neq 0 . .^{20}$

Firms are monopolistically competitive and engage in gods á la Calvo (1983). It is assumed that with probability $\left(1-\delta_{i}\right)$ firms in country $i$ are allowed to make optimal pricing decision. With probability $\delta_{i}$ firms are not allowed to change the price of their good. Firms maximize expected discounted stream of profits knowing that they need not be allowed to set their prices in each period. Optimal price for the firms that are allowed to change their price in period $t$, also known as the reset price in the literature, is denoted by $\mathfrak{P}_{i t}^{S}$. Firms' optimization problem can be written as

$$
\max _{\mathfrak{P}_{i t}^{S}} \mathrm{E}_{t}\left\{\sum_{k=0}^{\infty} \delta_{i}^{k} \mathfrak{M}_{i t, t+k} \Pi_{\ell, t+k}\left(\mathfrak{P}_{i t}^{S}\right)\right\} \text { for } \ell \in I_{i},
$$

where $\Pi_{\ell t}\left(\mathfrak{P}_{i t}^{S}\right)$ denotes profit of firm $\ell$ in period $t$ charging price $\mathfrak{P}_{i t}^{S}$. Firms maximize discounted stream of expected profits subject to the market clearing,

$$
\mathfrak{Y}_{t+k}(\ell)=\sum_{j=1}^{N} \frac{\mathfrak{C}_{j, t+k}(\ell)}{1-\tau_{j i}} \mathcal{P}_{j} \text { for } \ell \in I_{i} .
$$

Log-linearized first order condition to firms' optimization problem (A.25) and Calvo pricing law of motion for prices,

$$
p_{i t}^{d}=\delta_{i} p_{i t-1}^{d}+\left(1-\delta_{i}\right) \mathfrak{p}_{i t}^{s},
$$

imply that the dynamics of producer price inflation in terms of real marginal costs is described with the following familiar equation.

$$
\pi_{i t}^{d}=\beta \mathrm{E}_{t}\left\{\pi_{i, t+1}^{d}\right\}+\lambda_{i} \xi_{i t}
$$

where $\lambda_{i}=\left(1-\beta \delta_{i}\right)\left(1-\delta_{i}\right) / \delta_{i}, \pi_{i t}^{d}$ represents aggregate producer price inflation in country $i$ and $\xi_{i t}$ denotes log-deviations of real marginal costs (deflated by PPI) from steadystate value $\mu \equiv \ln \frac{\epsilon-1}{\epsilon}$,

$$
\xi_{i t}=w_{i t}-p_{i t}^{d}-a_{i t}-\mu
$$

\footnotetext{
${ }^{19}$ Concepts of weak and strong cross sectional dependence were introduced by Chudik, Pesaran, and Tosetti (2010).

${ }^{20}$ Single factor structure is assumed without the loss of generality. Analysis can be easily extended to accommodate multiple unobserved factors.
} 
A.1.3. Monetary Policy. Monetary policy in county $i$ is conducted by setting oneperiod risk-free nominal interest rate $r_{i t}$. Following Taylor-type monetary policy rules are assumed.

(A.28) $\quad r_{i t}=\phi_{\pi i} \mathrm{E}_{t} \pi_{i, t+1}+\phi_{\pi^{d} i} \pi_{i t}^{d}+\phi_{x i} x_{i t}-\ln \beta+\varepsilon_{\text {rit }}$, for $i \in\{1,2, \ldots, N\}$,

where $x_{i t}$ is an output gap in country $i$. $\varepsilon_{\text {rit }}$ is weakly cross-sectionally dependent $\operatorname{AR}(1)$ monetary policy shock,

$$
\varepsilon_{r i t}=\rho_{r i} \varepsilon_{r i, t-1}+\zeta_{\text {rit }}, \text { for } i \in\{1,2, \ldots, N\},
$$

where $\left|\rho_{r i}\right| \leq \rho<1$ and $\boldsymbol{\zeta}_{r t}=\left(\zeta_{r 1 t}, \ldots, \zeta_{r N t}\right)^{\prime}$ is a weakly cross sectionally dependent serially uncorrelated process with zero mean, $\mathrm{E}\left(\boldsymbol{\zeta}_{r t}\right)=\mathbf{0}$, and nonnegative definite covariance matrix $\mathrm{E}\left(\boldsymbol{\zeta}_{r t} \boldsymbol{\zeta}_{r t}^{\prime}\right)=\boldsymbol{\Sigma}_{r}$, such that $\varrho\left(\boldsymbol{\Sigma}_{r}\right)=O(1)$. Taylor rule (2.5) postulates that monetary authorities adjust interest rate according to the expected CPI inflation, current output gap and domestic producer price inflation. Furthermore, monetary policy shocks are possibly persistent and weakly cross sectionally dependent. Coefficients $\left\{\phi_{\pi_{i}}\right\},\left\{\phi_{\pi^{d_{i}}}\right\}$ and $\left\{\phi_{x i}\right\}$ are assumed to be bounded in $N$ in the asymptotic analysis of equilibrium. Alternative monetary policy rules could be investigated as well with some complications to the algebra. Determinacy of the equilibrium of' course depends on the set of monetary policy rules under consideration.

A.2. Market Clearing Conditions. Below we define the market clearing conditions for asset, goods and labor markets.

A.2.1. Market Clearing in Asset Markets. Assumption of complete asset markets implies that households share risk internationally, particularly, consumption indices across countries are connected as follows (see for instance Gali and Monacelli (2005))

$$
\frac{P_{i t}}{E_{i t}} C_{i t}=\frac{\varkappa_{i}}{\varkappa_{j}} C_{j t} \frac{P_{j t}}{E_{j t}}
$$

where constants $\varkappa_{i}, i \in\{1,2, \ldots, N\}$ with $\varkappa_{1}$ normalized to 1 , in general depend on initial conditions. Without the loss of generality, we shall assume the following symmetry condition

$$
\varkappa_{i}=1 \forall i \in\{1,2, \ldots, N\},
$$

in order to simplify the exposition. Simple expression (A.29) linking nominal spending expenditure of households is due to the logarithmic felicity function (A.1) in consumption. ${ }^{21}$

A.2.2. Market Clearing in Goods Markets. Market clearing in the goods markets implies that aggregate consumption of good $\ell$ must equal its production in the world economy, taking into account losses from shipping,

$$
\mathfrak{Y}_{t}(\ell)=\sum_{j=1}^{N} \Theta_{j i} \mathfrak{C}_{j t}(\ell) \mathcal{P}_{j} \quad \text { for } \ell \in I_{i} .
$$

Aggregate goods market clearing condition for goods produced in country $i$ can be written in nominal terms as

$$
\frac{P_{i t}^{d}}{E_{i t}} Y_{i t}=\sum_{j=1}^{N} \frac{P_{j i t}}{E_{j t}} C_{j i t} \mathcal{P}_{j},
$$

that is, nominal output of country $i$ equals nominal consumption of the country $i$ 's goods in the world economy (once converted into common currency). $Y_{i t}$ denotes the output index of country $i$, defined simply as

$$
Y_{i t} \equiv\left(\int_{I_{i}} \mathfrak{Y}_{t}^{\frac{\epsilon}{\epsilon-1}}(\ell) d \ell\right)^{\frac{\epsilon-1}{\epsilon}}
$$

\footnotetext{
${ }^{21}$ Market clearing for any asset a $\in \mathcal{S}$ with return $R_{j \text { at }}$ denominated in currency of country $j$ implies $\mathrm{E}_{t}\left\{\mathfrak{M}_{i t, t+1}\left(R_{j \mathrm{at}} \frac{E_{i j, t+1}}{E_{i j t}}\right)\right\}=1$. Equilibrium asset prices (including shares) can be computed from this households' optimality condition and the supply function of assets.
} 
Logarithm of output index $Y_{i t}$ and logarithm of aggregate real output $\mathfrak{Y}_{i t}=\int_{I_{i}} \mathfrak{Y}_{t}(\ell) d \ell$ are equal up to the first order. Denote the nominal share of country $i$ output that is exported to country $j$ as

$$
\chi_{i j t} \equiv \frac{P_{j i t} C_{j i t} \mathcal{P}_{j} E_{i t}}{P_{i t}^{d} Y_{i t} E_{j t}}
$$

If export shares $\chi_{i j t}$ are stationary, then the goods market clearing condition can be log-linearized around the steady state level of export shares, denoted as $\chi_{i j}$,

$$
y_{i t}=\sum_{j=1}^{N} \chi_{i j} c_{j i t}+\vartheta_{2 i},
$$

where constant $\vartheta_{2 i}=\sum_{j=1}^{N} \chi_{i j}\left(\theta_{j i}+\ln \mathcal{P}_{j}-\ln \chi_{i j}\right)$. Stationarity of export shares $\chi_{i j t}$ is established in Appendix A.4. Substituting demand function (A.12) into above expression yields relationship between output, consumption and producer price indices

$$
\mathbf{y}_{t}=\boldsymbol{\Upsilon} \mathbf{c}_{t}-\left(\mathbf{I}_{N}-\boldsymbol{\Upsilon} \boldsymbol{\Psi}\right)\left(\mathbf{p}_{t}^{d}-\mathbf{e}_{t}\right)+\boldsymbol{\vartheta}_{3}
$$

where $\Upsilon$ is matrix of steady-state nominal export shares with $(i, j)$-th coefficient being $\chi_{i j}$, and constant $\vartheta_{3 i}=\sum_{j=1}^{N}\left(\chi_{i j}\left(\ln \mathcal{P}_{j}+\ln \psi_{j i}-\ln \chi_{i j}\right)+\vartheta_{1 j}\right)$. Notice that only relative prices matter in the log-linearized market clearing condition (A.33), which is intuitive.

A.2.3. Market Clearing in Labor Markets. Market clearing in labor markets implies that demand for labor equals supply

$$
\int_{I_{i}} L_{t}(\ell) d \ell=L_{i t}=\stackrel{\circ}{L}_{i t} \mathcal{P}_{i}
$$

where supply of labor by a household in country $i, \stackrel{\circ}{L}_{i t}$, emanates from the intratemporal optimality condition (A.18).

A.3. Deriving a Canonical Representation of the Equilibrium. Equilibrium is solved approximately using traditional solution concept of log-linearization around steady-state. In a steady-state, all firms must charge the reset price $\mathfrak{P}_{i t}^{S}$, which has to be equal the optimal price mark-up and therefore the producer price index $P_{i t}^{d}$ reduces to:

$$
P_{i t}^{d}=\frac{W_{i t}}{A_{i t}} \frac{\epsilon}{\epsilon-1} \text {. }
$$

Using the fact that firms behave in a steady-state as if their price-setting was flexible, it is straightforward to solve for steady-state equilibrium. It is common in the literature on DSGE models to express deviations of marginal costs from the steady-state as a function of output gap and then to reduce the log-linearized system of equations characterizing equilibrium into NKPC, dynamic IS curve(s) and monetary policy rule(s); featuring three endogenous variables only: output gap, interest rates and inflation. ${ }^{22}$ Similar strategy is followed here.

A.3.1. New Keynesian Phillips Curves. Deviations of real marginal costs from a steady-state can be expressed as a function of aggregate output and productivity only.

$$
\begin{aligned}
\xi_{i t} & =w_{i t}-p_{i t}^{d}-a_{i t}-\mu, \\
& =\left(w_{i t}-p_{i t}\right)+\left(p_{i t}-p_{i t}^{d}\right)-a_{i t}-\mu, \\
& =\varphi i_{i t}+c_{i t}+p_{i t}-p_{i t}^{d}-a_{i t}-\mu, \\
& =\varphi y_{i t}+c_{i i t}-(1+\varphi) a_{i t}-\mu-\varphi \ln \mathcal{P}_{i}-\ln \psi_{i i},
\end{aligned}
$$

where the third equality substitutes household intratemporal optimality condition (A.18) and the last equality follows from household demand functions (A.12) and production function (A.22). Substituting solution for consumption of domestic goods $c_{i i t}$, equation (A.40) derived in Appendix, $\xi_{i t}$ reduces to

$$
\xi_{i t}=(1+\varphi) y_{i t}-(1+\varphi) a_{i t}+\vartheta_{6 i},
$$

\footnotetext{
${ }^{22}$ For example Gali and Monacelli (2005) and Chapter 4 of Woodford (2003).
} 
where constant $\vartheta_{6 i}=\ln \frac{\chi_{i i}}{\psi_{i i}}-\mu-(1+\varphi) \ln \mathcal{P}_{i}$. This expression allows us to define potential output, that is the level of output that would prevail if all prices were flexible,

$$
\bar{y}_{i t}=a_{i t}+\vartheta_{7 i},
$$

where $\vartheta_{7 i}=-(1+\varphi)^{-1} \vartheta_{6 i}$. Hence the NKPC is, using equation (A.26),

$$
\pi_{i t}^{d}=\beta \mathrm{E}_{t}\left\{\pi_{i, t+1}^{d}\right\}+\lambda_{i}(1+\varphi) x_{i t},
$$

where $x_{i t} \equiv y_{i t}-\bar{y}_{i t}$ is the output gap of country $i$.

A.3.2. Dynamic IS Equations (DIS). To derive version of a DIS equations, consumption $\mathbf{c}_{t}$ is expressed as a function of output gap $\mathbf{x}_{t}$ first. Using households' demand functions (A.12) and expression for consumption of domestic goods (A.40) derived in Appendix A.4 yields relationship between aggregate output, consumption and relative prices

$$
c_{i t}=y_{i t}-\left(p_{i t}-p_{i t}^{d}\right)-\vartheta_{8 i}
$$

where $\vartheta_{8 i}=\ln \sum_{k=1}^{N} \psi_{k i} \mathcal{P}_{k}$; or in matrix form, using equation (A.17),

$$
\mathbf{c}_{t}=\mathbf{y}_{t}+\left(\mathbf{I}_{N}-\boldsymbol{\Psi}\right)\left(\mathbf{p}_{t}^{d}-\mathbf{e}_{t}\right)-\boldsymbol{\vartheta}_{8}-\boldsymbol{\vartheta}_{1} .
$$

Substituting solution for relative prices (A.41) derived in Appendix A.4 allow us to write

$$
\mathrm{E}_{t} \Delta \mathbf{c}_{t+1}=\mathrm{E}_{t} \boldsymbol{\Psi} \Delta \mathbf{y}_{t+1}=\mathrm{E}_{t} \boldsymbol{\Psi} \Delta \mathbf{x}_{t+1}+\mathrm{E}_{t} \boldsymbol{\Psi} \Delta \mathbf{a}_{t+1} .
$$

Finally, substituting above expression for consumption into the households' intertemporal optimality condition (A.20) yields version of a dynamic IS curves

$$
\boldsymbol{\Psi} \mathbf{x}_{t}=\mathrm{E}_{t} \boldsymbol{\Psi} \mathbf{x}_{t+1}-\left(\mathbf{r}_{t}-\mathrm{E}_{t} \boldsymbol{\pi}_{t+1}+\ln \beta \cdot \boldsymbol{\tau}_{N}\right)+\mathrm{E}_{t} \boldsymbol{\Psi} \Delta \mathbf{a}_{t+1},
$$

where $\boldsymbol{\tau}_{N}$ is $N$-dimensional vector of ones. Productivity process (A.24) implies

$$
\mathrm{E}_{t} \boldsymbol{\Psi} \Delta \mathbf{a}_{t+1}=-\left(\mathbf{I}_{N}-\check{\boldsymbol{\rho}}_{a}\right) \varepsilon_{a t},
$$

where $\check{\boldsymbol{\rho}}_{a}$ is diagonal matrix with vector $\boldsymbol{\rho}_{a}$ on its diagonal; and equation (2.2) reduces to

$$
\boldsymbol{\Psi} \mathbf{x}_{t}=\mathrm{E}_{t} \boldsymbol{\Psi} \mathbf{x}_{t+1}-\left(\mathbf{r}_{t}-\mathrm{E}_{t} \boldsymbol{\pi}_{t+1}+\ln \beta \cdot \boldsymbol{\tau}_{N}\right)-\boldsymbol{\Psi}\left(\mathbf{I}_{N}-\check{\boldsymbol{\rho}}_{a}\right) \boldsymbol{\varepsilon}_{a t} .
$$

Note that innovations of the common technology factor, $\zeta_{f_{a} t}$, do not feature in (2.4) only because $f_{a t}$ is a martingale. Otherwise, $\zeta_{f_{a} t}$ would enter (2.4) as well.

\section{A.4. Steady-State Analysis and Derivations of Key Relations.}

A.4.1. Equilibrium Employment in a Steady-State. Substituting demand function (A.12), steady-state producer price (A.35) and production function (A.22) into market clearing for tradeable goods (A.30) yields

$$
\frac{\epsilon}{\epsilon-1} \frac{W_{i t}}{E_{i t}} L_{i t}=\sum_{j=1}^{N} \psi_{j i} \frac{P_{j t} C_{j t}}{E_{j t}} \mathcal{P}_{j} .
$$

Households' intratemporal optimality condition (A.18) can be written as

$$
\stackrel{\circ}{L}_{i t}^{1+\varphi}=\frac{W_{i t} \stackrel{\circ}{L}_{i t}}{P_{i t} C_{i t}}=\frac{W_{i t} L_{i t}}{P_{i t} C_{i t} \mathcal{P}_{i}} \Rightarrow W_{i t} L_{i t}=\stackrel{\circ}{L}_{i t}^{1+\varphi} P_{i t} C_{i t} \mathcal{P}_{i} .
$$

Substituting above expression for wage earnings $W_{i t} L_{i t}$ into (A.37) yields

$$
\stackrel{\circ}{L}_{i t}^{1+\varphi}=\frac{\epsilon-1}{\epsilon} \frac{\sum_{j=1}^{N} \psi_{j i} \frac{P_{j t} C_{j t}}{E_{j t}} \mathcal{P}_{j}}{\frac{P_{i t} C_{i t}}{E_{i t}} \mathcal{P}_{i}} .
$$

Finally, using risk sharing condition of households (A.29) allow us to write the following steady-state solution for individual employment level.

$$
\stackrel{\circ}{L}_{i}=\left(\frac{\epsilon-1}{\epsilon} \sum_{j=1}^{N} \psi_{j i} \frac{\mathcal{P}_{j}}{\mathcal{P}_{i}}\right)^{\frac{1}{1+\varphi}} .
$$


A.4.2. Equilibrium Relative Prices in A Steady-State. Intratemporal optimality condition for household in country $i$ and $j$ allows us to write relative wage as follows

$$
\frac{W_{i t} E_{j t}}{W_{j t} E_{i t}}=\left(\frac{\stackrel{\circ}{L}_{i t}}{\stackrel{\circ}{L}_{j t}}\right)^{\varphi} \frac{P_{i t} C_{i t}}{P_{j t} C_{j t}} \frac{E_{j t}}{E_{i t}} .
$$

Equilibrium relative wages in a steady-state can be solved by substituting solution for steady state employment $\stackrel{\circ}{L}_{i}$ (A.39) and risk sharing condition of households (A.29):

$$
\frac{W_{i t} E_{j t}}{W_{j t} E_{i t}}=\left(\frac{\stackrel{\circ}{L}_{i t}}{\stackrel{\circ}{L}_{j t}}\right)^{\varphi}=\left(\frac{\sum_{k=1}^{N} \psi_{k i} \mathcal{P}_{k}}{\sum_{k=1}^{N} \psi_{k j} \mathcal{P}_{k}} \frac{\mathcal{P}_{j}}{\mathcal{P}_{i}}\right)^{\frac{\varphi}{1+\varphi}} \equiv \frac{W_{i}^{r}}{W_{j}^{r}} .
$$

Having solved for steady-state equilibrium relative wages, it is easy to solve for equilibrium level of real exchange rate $q_{i j t}$ in a steady-state. By definition, $q_{i j t}$ is the logarithm of the relative consumer price indices between country $i$ and $j$ once converted into common currency:

$$
\begin{aligned}
q_{i j t} & =p_{i t}-p_{j t}+e_{j i t} \\
& =\sum_{k=1}^{N}\left(\psi_{j k}-\psi_{i k}\right) a_{k t}+\vartheta_{4 i j}
\end{aligned}
$$

where

$$
\vartheta_{4 i j}=\sum_{k=1}^{N}\left(\psi_{i k}-\psi_{j k}\right) \varphi i_{k}+\sum_{k=1}^{N}\left(\psi_{i k} \theta_{i k}-\psi_{j k} \theta_{j k}\right) .
$$

A.4.3. Steady-State Share of Nominal Exports on Output. Substituting demand function (A.12), steady-state producer price (A.35) and production function (A.22) into definition of export shares $\chi_{i j}$ (A.31) imply

$$
\chi_{i j}=\frac{\psi_{j i} P_{j t} C_{j t} \mathcal{P}_{j}}{\frac{\epsilon}{\epsilon-1} W_{i t} L_{i t}} \frac{E_{i t}}{E_{j t}},
$$

which reduces, after substituting expression (A.38) for wage earnings, international risk sharing condition of households (A.29) and solution for steady-state individual employment level $\stackrel{\circ}{i t}_{i t}$ A.39), to

$$
\chi_{i j}=\frac{\psi_{j i} \mathcal{P}_{j}}{\sum_{k=1}^{N} \psi_{k i} \mathcal{P}_{k}}
$$

A.4.4. Expression for Consumption of Domestic Goods $c_{i i t}$. Consumption of domestic goods $c_{i i t}$ is related to output in this subsection. Demand for goods (A.12) implies

$$
p_{i t}^{d}+c_{i i t}=\ln \psi_{i i}+p_{i t}+c_{i t},
$$

as well as

$$
p_{i t}^{d}+c_{j i t}+\theta_{j i}-e_{i t}+e_{j t}=\ln \psi_{j i}+p_{j t}+c_{j t} .
$$

Subtracting the above equations and substituting the risk sharing of households (A.29) yields

$$
c_{i i t}=c_{j i t}+\ln \frac{\psi_{i i}}{\psi_{j i}}+\theta_{j i}
$$

Substituting this expression into log-linearized goods market clearing condition (A.32) implies relationship between output and consumption of domestic good, $c_{i i t}$ :

$$
\begin{aligned}
y_{i t} & =\sum_{j=1}^{N} \chi_{i j}\left(c_{i i t}-\ln \frac{\psi_{i i}}{\psi_{j i}}-\theta_{j i}\right)+\vartheta_{2 i}, \\
& =c_{i i t}+\vartheta_{5 i},
\end{aligned}
$$

where $\vartheta_{5 i}=\ln \sum_{k=1}^{N} \psi_{k i} \mathcal{P}_{k}-\ln \psi_{i i}$. 
A.4.5. Relative Prices vs Relative Output. Subtracting the following two demand functions for domestic goods in country $i$ and $j$,

$$
\begin{aligned}
p_{i t}^{d}-e_{i t}+c_{i i t} & =\ln \psi_{i i}+p_{i t}-e_{i t}+c_{i t}, \\
p_{j t}^{d}-e_{j t}+c_{j j t} & =\ln \psi_{j j}+p_{j t}-e_{j t}+c_{j t},
\end{aligned}
$$

and using international risk sharing of households (A.29) yields

$$
c_{i i t}-c_{j j t}=\left(p_{j t}^{d}-e_{j t}\right)-\left(p_{i t}^{d}-e_{i t}\right)+\ln \frac{\psi_{i i}}{\psi_{j j}} .
$$

Substituting expression (A.40) for consumption of domestic goods implies relationship between relative producer prices and relative output:

$$
y_{i t}-y_{j t}=\left(p_{j t}^{d}-e_{j t}\right)-\left(p_{i t}^{d}-e_{i t}\right)+\vartheta_{8 i}-\vartheta_{8 j}
$$

where $\vartheta_{8, i}=\ln \sum_{k=1}^{N} \psi_{k i} \mathcal{P}_{k}$. Therefore

$$
-\left(\mathbf{I}_{N}-\mathbf{\Psi}\right)\left(\mathbf{p}_{t}^{d}-\mathbf{e}_{t}\right)=\left(\mathbf{I}_{N}-\mathbf{\Psi}\right) \mathbf{y}_{t}+\boldsymbol{\vartheta}_{9}
$$

where $\boldsymbol{\vartheta}_{9}=-\left(\mathbf{I}_{N}-\mathbf{\Psi}\right) \boldsymbol{\vartheta}_{8}$.

A.4.6. Relationship Between CPI and PPI Inflation. CPI-PPI relationship (A.17) implies

$$
\boldsymbol{\pi}_{t}-\boldsymbol{\pi}_{t}^{d}=\left(\boldsymbol{\pi}_{t}-\Delta \mathbf{e}_{t}\right)-\left(\boldsymbol{\pi}_{t}^{d}-\Delta \mathbf{e}_{t}\right)=-\left(\mathbf{I}_{N}-\boldsymbol{\Psi}\right)\left(\boldsymbol{\pi}_{t}^{d}-\Delta \mathbf{e}_{t}\right) .
$$

Substituting solution for relative prices (A.41) yields

$$
\boldsymbol{\pi}_{t}=\boldsymbol{\pi}_{t}^{d}+\left(\mathbf{I}_{N}-\boldsymbol{\Psi}\right) \Delta \mathbf{y}_{t}=\boldsymbol{\pi}_{t}^{d}+\left(\mathbf{I}_{N}-\boldsymbol{\Psi}\right)\left(\Delta \mathbf{x}_{t}+\Delta \mathbf{a}_{t}\right) .
$$




\begin{tabular}{|c|c|}
\hline \multicolumn{2}{|r|}{ Prices (Goods and Labor) } \\
\hline $\mathfrak{P}_{i t}(\ell)$ & Consumer price of good $\ell$ in country $i$ (and in currency of country $i$ ). \\
\hline $\mathfrak{P}_{t}^{d}(\ell)$ & Producer price of good $\ell$ (in domestic currency, i.e. country $j$ if $\ell \in I_{j}$ ). \\
\hline$P_{i t}$ & Consumer price index (minimal cost of purchasing one unit of $C_{i t}$ ). \\
\hline$P_{i j t}$ & CPI index of goods imported from country $j$ for the household in country $i$. \\
\hline$P_{i t}^{d}$ & Producer price index of country $i$. \\
\hline$W_{i t}$ & Wage earned by household in country $i$ per one unit of supplied labor. \\
\hline$\pi_{i t}, \pi_{i t}^{d}$ & CPI and PPI inflation in country $i$, respectively. \\
\hline \multicolumn{2}{|r|}{ Consumption } \\
\hline $\mathfrak{C}_{i t}(\ell)$ & Consumption of good $\ell$ by a household in country $i$. \\
\hline$C_{i t}$ & Consumption index of a household in country $i$. \\
\hline \multirow[t]{2}{*}{$C_{i j t}$} & Consumption index of tradeable goods imported from country $j$ and consumed by \\
\hline & household in country $i$. \\
\hline \multicolumn{2}{|r|}{ Employment and Population } \\
\hline$\dot{L}_{i t}, L_{i t}$ & Employment per capita and aggregate employment (hours) in country $i$, respectively. \\
\hline$L_{t}(\ell)$ & Employment in firm $\ell$. \\
\hline $\mathcal{P}_{i}$ & Population in country $i$. \\
\hline \multicolumn{2}{|r|}{ Output } \\
\hline $\mathfrak{Y}_{i t}, Y_{i t}$ & Real output and real output index of country $i$, respectively. \\
\hline $\mathfrak{Y}_{t}(\ell)$ & Real output of firm $\ell$ in period $t$ (units). \\
\hline \multicolumn{2}{|r|}{ Other symbols and variables used } \\
\hline $\mathfrak{M}_{i t, t+1}$ & Stochastic discount factor of a household in country $i$ between period $t$ and $t+1$. \\
\hline$A_{i t}$ & Productivity in country $i$. \\
\hline$Q_{i j t}$ & CPI based RER between country $i$ and $j$. \\
\hline$R_{i t}$ & Gross return on one period bond (policy instrument). $r_{i t}=\ln \left(R_{i t}\right)$ \\
\hline$E_{i t}$ & The amount of currency $i$ per one unit of numeraire currency 1. $E_{i j t}=E_{i t} / E_{j t}$ \\
\hline \multirow[t]{2}{*}{$\chi_{i j},\left(\psi_{j i}\right)$} & Steady-state share of nominal exports (imports) from country $i$ to country $j$ on \\
\hline & nominal GDP (consumption) of country $i$. \\
\hline$\xi_{i t}$ & Log-deviation of real marginal cost (deflated by PPI) from a steady-state. \\
\hline$\Pi_{\ell t}$ & Profit of firm $\ell$ (in domestic currency). \\
\hline$\Upsilon, \Psi$ & Matrix of steady-state export shares and import shares, respectively. \\
\hline $\mathrm{E}_{t}$ & Expectation operator (conditional on the information at time $t$ ). \\
\hline$\Theta_{i j} \equiv \frac{1}{1-\tau_{i j}}$ & One plus the tariff equivalent of iceberg shipping $\operatorname{costs} \tau_{i j}$ \\
\hline
\end{tabular}

TABLE 1: List of Main Variables and Symbols 


\section{Appendix B. Proofs, Definitions and Additional Lemmas}

Lemma 3. Let $\mathbf{A}_{n}=\mathbf{\Psi}_{n}-\mathbf{I}_{n}$ where $n \geq 1$ and $\mathbf{\Psi}_{n}$ is $n \times n$ import share matrix, that is

$$
\boldsymbol{\Psi}_{n} \geq 0 \text { and } \boldsymbol{\Psi}_{n} \boldsymbol{\tau}_{n}=\boldsymbol{\tau}_{n} .
$$

If no country or group of countries is isolated in the region of $n$ countries characterized by import share matrix $\boldsymbol{\Psi}_{n}$, then

$$
\operatorname{rank}\left(\mathbf{A}_{n}\right)=n-1
$$

Proof. Proof is by induction.

(1) In the first step of induction (the basis), we show that Lemma 3 holds for $n=1$. This case is trivial. If $n=1$, then $\boldsymbol{\Psi}_{1}=1, \mathbf{A}_{1}=0$ and $\operatorname{rank}\left(\mathbf{A}_{1}\right)=\operatorname{rank}(0)=$ 0.

(2) In the second step of induction (the inductive step), we show that if Lemma 3 holds for $n=m$ countries, then it holds for $n=m+1$ countries. Consider any matrix $\mathbf{A}_{m+1}=\Psi_{m+1}-\mathbf{I}_{m+1}$ where $\boldsymbol{\Psi}_{m+1}$ is import share matrix. If no country or group of countries is isolated in the region of $m+1$ countries characterized by import share matrix $\boldsymbol{\Psi}_{m+1}$, then at least one diagonal element of matrix $\mathbf{A}_{m+1}$ is negative (otherwise region would consist of $m+1 \geq 2$ isolated countries). Suppose, without the loss generality, it is the last diagonal element, $a_{m+1, m+1}^{(m+1)} \cdot{ }^{23}$ Now perform the following row operations on the matrix $\mathbf{A}_{m+1}$. For $i \in\{1,2, \ldots, m\}$, multiply the last row of matrix $\mathbf{A}_{m+1}$ by scalar $-a_{i, m+1}^{(m+1)} / a_{m+1, m+1}^{(m+1)}$ and add the product (row-vector) to the row $i$ of matrix $\mathbf{A}_{m+1}$. Resulting matrix, denoted as $\mathbf{C}_{m+1}$, can be written as

$$
\mathbf{C}_{m+1}=\left(\begin{array}{cc}
\mathbf{A}_{m} & \mathbf{0}_{m} \\
a_{m+1,1}^{(m+1)}, \ldots, a_{m, 1}^{(m+1)} & a_{m+1, m+1}^{(m+1)}
\end{array}\right)
$$

where $(i, j)$-th element of matrix $\mathbf{A}_{m}$, denoted as $a_{i j}^{(m)}$, is

$$
a_{i j}^{(m)}=a_{i j}^{(m+1)}-a_{m+1, j}^{(m+1)} \frac{a_{i, m+1}^{(m+1)}}{a_{m+1, m+1}^{(m+1)}} .
$$

It is clear that $\operatorname{rank}(\mathbf{C})=\operatorname{rank}\left(\mathbf{A}_{m+1}\right)$. Matrix $\mathbf{A}_{m}$ satisfies

$$
\mathbf{A}_{m} \boldsymbol{\tau}_{m}=0, a_{i i}^{(m)} \leq 0 \text { and for } i \neq j: a_{i j}^{(m)} \geq 0
$$

and the matrix $\mathbf{\Psi}_{m}=\mathbf{A}_{m}+\mathbf{I}_{m}$ is indeed an import share matrix. Furthermore, the division of $m+1$ countries into isolated subgroups of countries is unchanged by the performed row-operations. This is because the countries that previously (in the matrix $\mathbf{A}_{m+1}$ ) imported goods from country $m+1$ are now (in the new matrix $\mathbf{C}_{m+1}$ ) directly importing goods from the trade partners of country $m+1$. By induction hypothesis, $\operatorname{rank}\left(\mathbf{A}_{m}\right)=m-1$. Since also $a_{m+1, m+1}^{(m+1)}<0$, it follows

$$
\operatorname{rank}\left(\mathbf{A}_{m+1}\right)=\operatorname{rank}(\mathbf{C})=1+\operatorname{rank}\left(\mathbf{A}_{m}\right)=m .
$$

This completes the proof.

Definition 4. The directed graph of $N \times N$ dimensional matrix $\mathbf{A}_{N}$, denoted by $\Gamma\left(\mathbf{A}_{N}\right)$ is the directed graph on $N$ nodes $T_{1}, T_{2}, \ldots, T_{N}$ such that here is a directed arc in $\Gamma\left(\mathbf{A}_{N}\right)$ from $T_{i}$ to $T_{j}$ if and only if $a_{N} i j \neq 0$.

Definition 5. A directed path $\gamma$ in a graph $\Gamma$ is a sequence of arcs $T_{i_{1}} T_{i_{2}}, T_{i_{2}} T_{i_{3}}, \ldots$ in $\Gamma$. The length of a directed path is the number of successive arcs in the directed path if this number is finite; otherwise, the directed path is said to have infinite length.

\footnotetext{
${ }^{23}$ We denote the $(i, j)$-th element of matrix $\mathbf{A}_{k}$ as $a_{i j}^{(k)}$.
} 
Following lemma establishes the order of magnitudes of the sum, the product and the inverse of certain matrices.

Lemma 4. Let $\mathcal{K}$ denote a set of $K$ natural numbers, $K$ is fixed, and let $\mathbf{A}_{N}, \mathbf{B}_{N}$ denote arbitrary $N \times N$ dimensional matrices that satisfy

$$
a_{N} i j=\left\{\begin{array}{cl}
\alpha_{i j}+o(1) & (i, j) \in \mathcal{Z}_{\mathbf{A}_{N}} \\
o(1) & i \neq j \wedge j \in \mathcal{K} \\
O\left(N^{-1}\right) & i \neq j \wedge j \notin \mathcal{K}
\end{array} \quad, \quad b_{N} i j=\left\{\begin{array}{cc}
\beta_{i j}+o(1) & (i, j) \in \mathcal{Z}_{\mathbf{B}_{N}} \\
o(1) & i \neq j \wedge j \in \mathcal{K} \\
O\left(N^{-1}\right) & i \neq j \wedge j \notin \mathcal{K}
\end{array} .\right.\right.
$$

where $\left|\alpha_{i j}\right|<M,\left|\beta_{i j}\right|<M$ and set $\mathcal{Z}_{\mathbf{A}_{N}}\left(\mathcal{Z}_{\mathbf{B}_{N}}\right)$ includes coordinates of all diagonal elements $(i, i), i=1, . ., N$, and bounded number $Z_{\mathbf{A}}\left(Z_{\mathbf{B}}\right)$ in $N$ of off-diagonal elements of matrix $\mathbf{A}_{N}\left(\mathbf{B}_{N}\right)$. Let $\mathbf{Z}_{A_{N}}$ be $N \times N$ dimensional matrix such that $\left[\mathbf{Z}_{A_{N}}\right]_{i j}=1$ if $(i, j) \in \mathcal{Z}_{\mathbf{A}_{N}}$ and zero elsewhere. Define following sets

$$
\begin{aligned}
\mathcal{C}\left(\mathbf{A}_{N}, \mathbf{B}_{N}\right) & =\left\{(i, j) ; \exists \ell \in\{1, ., N\}:(i, \ell) \in \mathcal{Z}_{\mathbf{A}_{N}} \wedge(\ell, j) \in \mathcal{Z}_{\mathbf{B}_{N}}\right\}, \\
\mathcal{L}_{i j} & =\left\{1 \leq \ell \leq N ;(i, \ell) \in \mathcal{Z}_{\mathbf{A}_{N}} \wedge(\ell, j) \in \mathcal{Z}_{\mathbf{B}_{N}}\right\} .
\end{aligned}
$$

Then following statements hold.

(1)

$$
\left[\mathbf{A}_{N} \mathbf{B}_{N}\right]_{i j}=\left\{\begin{array}{cc}
\sum_{\mathcal{L}_{i j}} \alpha_{i \ell} \beta_{\ell j}+o(1) & (i, j) \in \mathcal{C}\left(\mathbf{A}_{N}, \mathbf{B}_{N}\right) \\
o(1) & (i, j) \notin \mathcal{C}\left(\mathbf{A}_{N}, \mathbf{B}_{N}\right) \wedge j \in \mathcal{K} \cup \mathcal{S}\left(\mathbf{B}_{N}\right) \\
O\left(N^{-1}\right) & (i, j) \notin \mathcal{C}\left(\mathbf{A}_{N}, \mathbf{B}_{N}\right) \wedge j \notin \mathcal{K} \cup \mathcal{S}\left(\mathbf{B}_{N}\right)
\end{array},\right.
$$

where set $\mathcal{S}\left(\mathbf{B}_{N}\right) \equiv\left\{\ell \in\{1,2, \ldots, N\} ; \exists k \leq N:(k, \ell) \in \mathcal{Z}_{\mathbf{B}_{N}} \wedge k \in \mathcal{K}\right\}$ has fi(2)

$$
\left[\mathbf{A}_{N}+\mathbf{B}_{N}\right]_{i j}=\left\{\begin{array}{cc}
\alpha_{i j}+o(1) & (i, j) \in \mathcal{Z}_{\mathbf{A}_{N} \backslash \mathcal{Z}_{\mathbf{B}_{N}}} \\
\beta_{i j}+o(1) & (i, j) \in \mathcal{Z}_{\mathbf{B}_{N} \backslash \mathcal{Z}_{\mathbf{A}_{N}}} \\
\alpha_{i j}+\beta_{i j}+o(1) & (i, j) \in \mathcal{Z}_{\mathbf{A}_{N}} \cap \mathcal{Z}_{\mathbf{B}_{N}} \\
o(1) & (i, j) \notin \mathcal{Z}_{\mathbf{A}_{N} \cup \mathcal{Z}_{\mathbf{B}_{N}} \wedge j \in \mathcal{K}} \\
O\left(N^{-1}\right) & (i, j) \notin \mathcal{Z}_{\mathbf{A}_{N}} \cup \mathcal{Z}_{\mathbf{B}_{N}} \wedge j \notin \mathcal{K}
\end{array}\right.
$$

(3) Furthermore, if $\mathbf{A}_{N}$ also satisfies

$$
\lim _{N \rightarrow \infty} \min _{1 \leq i \leq N}\left\{\left|a_{N} i i\right|-\sum_{j=1, j \neq i}^{N}\left|a_{N} i j\right|\right\}=c>0
$$

then

$$
\left[\mathbf{A}_{N}^{-1}\right]_{i j}=\left\{\begin{array}{cc}
g_{i j}+o(1) & (i, j) \in \mathcal{A}_{N} \\
o(1) & (i, j) \notin \mathcal{A}_{N} \wedge j \in \mathcal{B}_{N} \\
O\left(N^{-1}\right) & (i, j) \notin \mathcal{A}_{N} \wedge j \notin \mathcal{B}_{N}
\end{array}\right.
$$

where set $\mathcal{A}_{N}=\bigcup_{k=1}^{N} \mathcal{C}\left(\mathbf{A}_{N}^{k}, \mathbf{A}_{N}\right)=\left\{(i, j) \in\{1, . ., N\} \times\{1, . ., N\} ;\left[\mathbf{Z}_{A_{N}}^{N}\right]_{i j}=1\right\}$, equivalently $(i, j) \in \mathcal{A}_{N} \Leftrightarrow \exists$ a directed path $\gamma_{i j}$ in the directed graph $\Gamma\left(\mathbf{Z}_{A_{N}}\right)$ connecting nodes $T_{i}$ and $T_{j}$ of $\Gamma\left(\mathbf{Z}_{A_{N}}\right)$. Set $\mathcal{B}_{N}=\bigcup_{k=1}^{N} \mathcal{S}\left(\mathbf{A}_{N}^{k}\right) \cup \mathcal{K}=\mathcal{K} \cup$ $\left\{j \in\{1, . ., N\} ; \exists i \in\{1, . ., N\}: i \neq j \wedge(i, j) \in \mathcal{A}_{N}\right\}$ has bounded number of elements in $N . g_{i j}$ is a function of coefficients $\left\{\alpha_{i k} ; k \in \mathcal{R}_{i}\right\}$ in general, where $\mathcal{R}_{i} \equiv\left\{1 \leq k \leq N ; \exists\right.$ directed path $\gamma_{i k}$ connecting nodes $T_{i}$ and $T_{k}$ of $\left.\Gamma\left(\mathbf{Z}_{A_{N}}\right)\right\}$.

Proof. Define $\mathcal{H}_{i j} \equiv\left\{1 \leq k \leq N ;(i, k) \in \mathcal{Z}_{\mathbf{A}_{N}} \vee(k, j) \in \mathcal{Z}_{\mathbf{B}_{N}}\right\}$ and $\mathcal{D}_{i j} \equiv\left\{1 \leq k \leq N ; k \in \mathcal{K} \cup \mathcal{H}_{i j} \backslash \mathcal{L}_{i j}\right\}$. Set $\mathcal{D}_{i j}$ has fixed number of elements (as $\left.N \rightarrow \infty\right)$. For $(i, j) \in \mathcal{C}\left(\mathbf{A}_{N}, \mathbf{B}_{N}\right)$ :

$\left[\mathbf{A}_{N} \mathbf{B}_{N}\right]_{i j}=\sum_{k \in \mathcal{L}_{i j}} \underbrace{a_{N i k} b_{N k j}}_{\alpha_{i \ell} \beta_{\ell j}+o(1)}+\sum_{\substack{k=1 \\ k \notin \mathcal{D}_{i j}, k \notin \mathcal{L}}}^{N} \underbrace{a_{N i k} b_{N k j}}_{o\left(N^{-1}\right)}+\sum_{k \in \mathcal{D}_{i j}} \underbrace{a_{N i k} b_{N} k j}_{o(1)}=\sum_{k \in \mathcal{L}_{i j}} \alpha_{i \ell} \beta_{\ell j}+o(1)$. 
For $(i, j) \notin \mathcal{C}\left(\mathbf{A}_{N}, \mathbf{B}_{N}\right):$

$\left[\mathbf{A}_{N} \mathbf{B}_{N}\right]_{i j}=\left\{\begin{array}{cc}\sum_{k \in \mathcal{H}_{i j}} \underbrace{a_{N i k} b_{N k j}}_{o(1)}+\sum_{\substack{k=1 \\ k \notin \mathcal{D}}}^{N} \underbrace{a_{N i k} b_{N k j}}_{o\left(N^{-1}\right)}+\sum_{k \in \mathcal{K} \backslash \mathcal{H}_{i j}} \underbrace{a_{N^{i k}} b_{N} k j}_{o(1)}=o(1) & j \in \mathcal{K} \\ \sum_{k \in \mathcal{H}_{i j}} \underbrace{a_{N i k} b_{N k j}}_{O\left(N^{-1}\right)}+\sum_{\substack{k=1 \\ k \notin \mathcal{D}_{i j}}}^{a_{O\left(N^{-2}\right)}^{a_{N i k} b_{N k j}}}+\sum_{k \in \mathcal{K} \backslash \mathcal{H}_{i j}} \underbrace{a_{N^{i k}} b_{N k j}}_{o\left(N^{-1}\right)}=O\left(N^{-1}\right) \quad j \notin \mathcal{K} \cup \mathcal{S}\left(\mathbf{B}_{N}\right)\end{array}\right.$.

Finally for $(i, j) \notin \mathcal{C}\left(\mathbf{A}_{N}, \mathbf{B}_{N}\right)$ and $j \in \mathcal{S}\left(\mathbf{B}_{N}\right) \backslash \mathcal{K}$, order of magnitude of the element $\left[\mathbf{A}_{N} \mathbf{B}_{N}\right]_{i j}$ is:

$\sum_{k:(i, k) \in \mathcal{Z}_{\mathbf{A}_{N}}} \underbrace{a_{N i k} b_{N} k j}_{O\left(N^{-1}\right)}+\sum_{k:(k, j) \in \mathcal{Z}_{\mathbf{B}_{N}}} \underbrace{a_{N i k} b_{N} k j}_{o(1)}+\sum_{\substack{k=1 \\ k \notin \mathcal{D}_{i j}}}^{N} \underbrace{a_{N i k} b_{N} k j}_{O\left(N^{-2}\right)}+\sum_{k \in \mathcal{K} \backslash \mathcal{H}_{i j}} \underbrace{a_{N} i k b_{N} k j}_{o\left(N^{-1}\right)}=o(1)$.

This proves (B.4). Establishing the order of magnitudes of matrix $\mathbf{A}_{N}+\mathbf{B}_{N}$ (equation (B.5)) is straightforward by noting that

$$
\left[\mathbf{A}_{N}+\mathbf{B}_{N}\right]_{i j}=a_{N} i j+b_{N} i j
$$

Assumption (B.6) implies that for $0<\delta<c$,

$$
\exists N_{0} \in \mathbb{N} ; \forall N>N_{0}: a_{N i i}>\sum_{j=1, j \neq i}^{N}\left|a_{N} i j\right|
$$

That is, matrix $\mathbf{A}_{N}$ is strictly diagonally dominant for any $N>N_{0}$. Let $\check{\mathbf{D}}_{N}$ denote diagonal matrix with elements $a_{N} i i>0$ on its diagonal. Matrix

$$
\mathbf{G}_{N} \equiv \mathbf{I}_{N}-\mathbf{D}_{N}^{-1} \mathbf{A}_{N}
$$

satisfies $\left\|\mathbf{G}_{N}\right\|_{r}<1$, which implies $\varrho\left(\mathbf{G}_{N}\right)<1$. Matrix $\mathbf{I}_{N}-\mathbf{G}_{N}$ is invertible. To see this, note that $\lim _{k \rightarrow \infty}\left\|\mathbf{G}_{N}^{k}\right\|_{r}=0$ and

$$
\left(\mathbf{I}_{N}-\mathbf{G}_{N}\right) \sum_{k=0}^{\ell} \mathbf{G}_{N}^{k}=\sum_{k=0}^{\ell} \mathbf{G}_{N}^{k}-\sum_{k=1}^{\ell+1} \mathbf{G}_{N}^{k}=\mathbf{I}_{N}-\mathbf{G}_{N}^{\ell+1} .
$$

Hence $\left(\mathbf{I}_{N}-\mathbf{G}_{N}\right)^{-1}=\sum_{k=0}^{\infty} \mathbf{G}_{N}^{k}$. Inverse of matrix $\mathbf{D}_{N}^{-1} \mathbf{A}_{N}=\mathbf{I}_{N}-\mathbf{G}_{N}$ is $\sum_{k=0}^{\infty} \mathbf{G}_{N}^{k}$. Therefore for any $N>N_{0}, \mathbf{A}_{N}^{-1}$ exists and it is given by

$$
\mathbf{A}_{N}^{-1}=\sum_{k=0}^{\infty} \mathbf{G}_{N}^{k} \mathbf{D}_{N}^{-1}=\mathbf{D}_{N}^{-1}+\sum_{k=1}^{\infty} \mathbf{G}_{N}^{k} \mathbf{D}_{N}^{-1}
$$

Equations (B.4), (B.5) and (B.8) establish (B.7).

LEMMA 5. Let $\left\{\varepsilon_{i t}\right\}$ be generated according to the following weakly cross-sectionally dependent stationary $A R(1)$ process:

$$
\varepsilon_{i t}=\rho_{i} \varepsilon_{i t-1}+\zeta_{i t} \quad \text { for } i=1,2, \ldots, N,
$$

where $\left|\rho_{i}\right|<\rho<1$, disturbances collected into vector $\boldsymbol{\zeta}_{t}=\left(\zeta_{1 t}, \ldots, \zeta_{N t}\right)^{\prime}$ are serially uncorrelated, $E\left(\boldsymbol{\zeta}_{t}\right)=0$, and $E\left(\boldsymbol{\zeta}_{t} \boldsymbol{\zeta}_{t}^{\prime}\right)=\boldsymbol{\Sigma}_{\zeta}$ with bounded maximum eigenvalue in $N$, $\varrho\left(\boldsymbol{\Sigma}_{\zeta}\right)=O(1)$. Let $\boldsymbol{\omega}=\left(\omega_{1}, \ldots, \omega_{N}\right)^{\prime}$ be pre-determined weights satisfying

$$
\boldsymbol{\omega}^{\prime} \boldsymbol{\omega}=o(1)
$$

Then

$$
\omega^{\prime} \zeta_{t} \stackrel{q . m .}{\rightarrow} 0, \text { as } N \rightarrow \infty
$$


Proof. $\mathrm{E}\left(\boldsymbol{\omega}^{\prime} \boldsymbol{\zeta}_{t}\right)=\boldsymbol{\omega}^{\prime} \mathrm{E}\left(\boldsymbol{\zeta}_{t}\right)=0$. Let $\check{\boldsymbol{\rho}}$ be diagonal matrix with vector $\left(\rho_{1}, \rho_{2}, \ldots, \rho_{N}\right)^{\prime}$ on its diagonal.

$$
\begin{aligned}
\operatorname{Var}\left(\boldsymbol{\omega}^{\prime} \boldsymbol{\zeta}_{t}\right) & =\operatorname{Var}\left(\sum_{k=0}^{\infty} \boldsymbol{\omega}^{\prime} \check{\boldsymbol{\rho}}^{k} \boldsymbol{\zeta}_{t-k}\right)=\sum_{k=0}^{\infty} \boldsymbol{\omega}^{\prime} \check{\boldsymbol{\rho}}^{k} \boldsymbol{\Sigma}_{\zeta} \check{\boldsymbol{\rho}}^{k} \boldsymbol{\omega} \\
& \leq \varrho\left(\boldsymbol{\Sigma}_{\zeta}\right) \sum_{k=0}^{\infty} \boldsymbol{\omega}^{\prime} \check{\boldsymbol{\rho}}^{2 k} \boldsymbol{\omega} \leq \varrho\left(\boldsymbol{\Sigma}_{\zeta}\right) \underbrace{\boldsymbol{\omega}^{\prime} \boldsymbol{\omega}}_{o(1)} \sum_{k=0}^{\infty} \underbrace{\varrho\left(\check{\boldsymbol{\rho}}^{2 k}\right)}_{<\rho^{2 k}}, \\
& =o(1) .
\end{aligned}
$$

It follows $\boldsymbol{\omega}^{\prime} \boldsymbol{\zeta}_{t} \stackrel{q . m .}{\rightarrow} 0$, as $N \rightarrow \infty$.

Lemma 6. Let $\mathcal{K}$ denote a set of $K$ natural numbers, $K$ is fixed, and let $\mathbf{A}_{N}, \mathbf{B}_{N}$ denote arbitrary $N \times N$ dimensional matrices such that

$$
a_{N} i j=\left\{\begin{array}{cl}
\alpha_{i j}+o(1) & i=j \vee j \in \mathcal{K} \\
O\left(N^{-1}\right) & i \neq j \wedge j \notin \mathcal{K}
\end{array} \quad b_{N i j}=\left\{\begin{array}{cl}
\beta_{i j}+o(1) & i=j \vee j \in \mathcal{K} \\
O\left(N^{-1}\right) & i \neq j \wedge j \notin \mathcal{K}
\end{array},\right.\right.
$$

where $\left|\alpha_{i j}\right|<M,\left|\beta_{i j}\right|<M$. Then

$$
\left[\mathbf{A}_{N} \mathbf{B}_{N}\right]_{i j}=\left\{\begin{array}{cl}
O(1) & i=j \vee j \in \mathcal{K} \\
O\left(N^{-1}\right) & i \neq j \wedge j \notin \mathcal{K}
\end{array},\right.
$$

and

$$
\left[\mathbf{A}_{N}+\mathbf{B}_{N}\right]_{i j}=\left\{\begin{array}{cl}
\alpha_{i j}+\beta_{i j}+o(1) & i=j \vee j \in \mathcal{K} \\
O\left(N^{-1}\right) & i \neq j \wedge j \notin \mathcal{K}
\end{array} .\right.
$$

Furthermore, if $\mathbf{A}_{N}$ also satisfies

$$
\lim _{N \rightarrow \infty} \min _{1 \leq i \leq N}\left\{\left|a_{N i i}\right|-\sum_{j=1, j \neq i}^{N}\left|a_{N i j}\right|\right\}=c>0
$$

then

$$
\left[\mathbf{A}_{N}^{-1}\right]_{i j}=\left\{\begin{array}{cl}
O(1) & i=j \vee j \in \mathcal{K} \\
O\left(N^{-1}\right) & i \neq j \wedge j \notin \mathcal{K}
\end{array}\right.
$$

Proof.

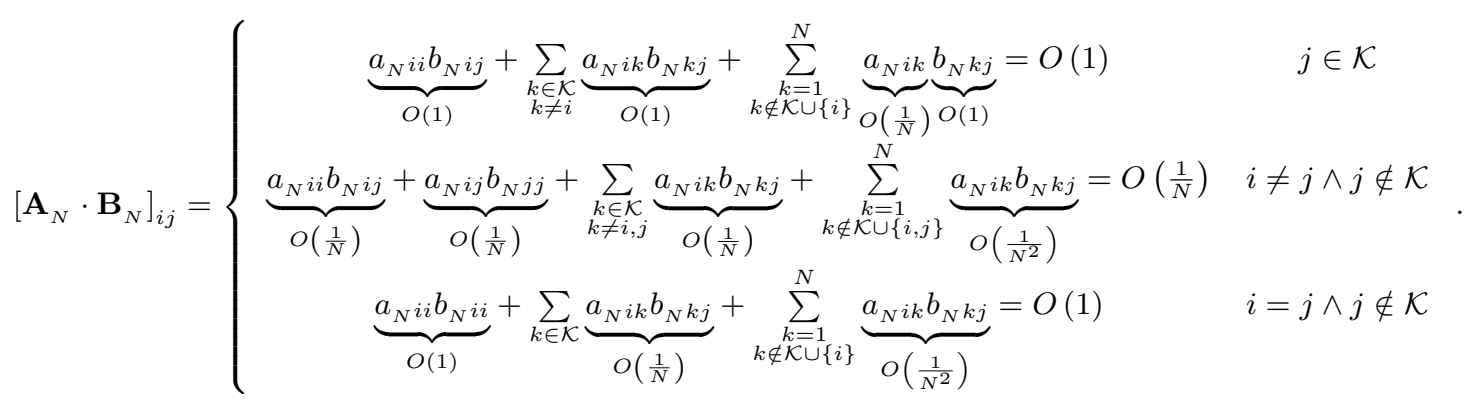

$$
[\mathbf{A}+\mathbf{B}]_{i j}=a_{N i j}+b_{N} i j=\left\{\begin{array}{cc}
\alpha_{i j}+\beta_{i j}+o(1) & i=j \vee j \in \mathcal{K} \\
O\left(N^{-1}\right) & i \neq j \wedge j \notin \mathcal{K}
\end{array}\right.
$$

This proves results (B.12) and (B.13).

Proof for the order of magnitude of the elements of $\mathbf{A}_{N}^{-1}$ is identical to the proof of Lemma 4, equation (B.7), taking into account equations (B.12) and (B.13). 


\section{B.1. Proof of Lemma 2.}

\section{Proof (Lemma 2).}

a. $(\Rightarrow)$ Denote matrix $\Psi-\mathbf{I}_{N} \equiv$ B. $K$ isolated groups of countries imply that there exist indexation of countries for which matrix $\mathbf{B}$ is the following block diagonal matrix.

$$
\mathbf{B}=\left(\begin{array}{cccc}
\mathbf{B}_{1} & \mathbf{0} & \cdots & \mathbf{0} \\
\mathbf{0} & \mathbf{B}_{2} & & \mathbf{0} \\
\vdots & & \ddots & \vdots \\
\mathbf{0} & \mathbf{0} & \cdots & \mathbf{B}_{K}
\end{array}\right)
$$

Denote the number of columns of (square) matrix $\mathbf{B}_{i}$ by $n_{i}$. Note that $\mathbf{B}_{i}+\mathbf{I}_{n_{i}}$ satisfies equivalent conditions to (A.3) for any $i \in\{1,2, \ldots, K\}$, particularly

$$
\mathbf{B}_{i} \boldsymbol{\tau}_{n_{i}}=\mathbf{0}_{n_{i}} \text {, and } \mathbf{B}_{i}+\mathbf{I}_{n_{i}} \geq 0 \text {. }
$$

It follows that

$$
\operatorname{rank}\left(\mathbf{B}_{i}\right) \leq n_{i}-1
$$

The group of countries corresponding to matrix $\mathbf{B}_{i}$ forms one trade region (i.e. no subgroup of countries is isolated within the group). Therefore, it follows from Lemma 3 that

$$
\operatorname{rank}\left(\mathbf{B}_{i}\right)=n_{i}-1
$$

Since $\sum_{i=1}^{K} n_{i}=N$ by construction, we have

$$
\operatorname{rank}(\mathbf{B})=\sum_{i-1}^{K} \operatorname{rank}\left(\mathbf{B}_{i}\right)=N-K
$$

b. $(\Leftarrow)$ (By contradiction). Assume $\operatorname{rank}\left(\mathbf{I}_{N}-\Psi\right)=N-K$ and world consists of $K_{a} \neq$ $K$ isolated trade regions, where $K \geq 1$ and $K_{a} \geq 1$. Then, using part (a) of the proof, assumption of $K_{a}$ isolated trade regions implies $N-\operatorname{rank}\left(\mathbf{I}_{N}-\mathbf{\Psi}\right)=$ $K_{a} \neq K$. This contradicts assumption $\operatorname{rank}\left(\mathbf{I}_{N}-\mathbf{\Psi}\right)=N-K$.

\section{B.2. Proof of Lemma 1.}

Proof (Lemma 1). Equilibrium is determinate if and only if all eigenvalues of $\mathbf{A}$ lie inside the unit circle, i.e. the spectral radius of $\mathbf{A}$, denoted as $\varrho(\mathbf{A})$, is less than one. Sufficient condition for $\varrho(\mathbf{A})<1$ is $\|\mathbf{A}\|_{M}<1$, where we use $\|\cdot\|_{M}$ to denote any matrix norm. ${ }^{24}$ A particular convenient matrix norm is

$$
\|\mathbf{A}\|_{r} \equiv \max _{1 \leq k \leq N} \sum_{n=1}^{N}\left|a_{k n}\right| .
$$

We are going to find the set of monetary policy rules for which $\|\mathbf{A}\|_{r}<1$. Notice that matrix $\mathbf{A}=\mathbf{A}_{0}^{-1} \mathbf{A}_{1}$ is $^{25}$

$$
\mathbf{A}=\left(\begin{array}{cc}
\beta \check{\boldsymbol{\phi}}_{\pi^{d}}^{-1}\left(\boldsymbol{\Xi}+\check{\boldsymbol{\phi}}_{x}\right) \mathbf{H}^{-1} \check{\boldsymbol{\phi}}_{\pi^{d}}+(1+\varphi) \check{\boldsymbol{\lambda}} \mathbf{H}^{-1}\left(\mathbf{I}_{N}-\check{\boldsymbol{\phi}}_{\pi}\right) & (1+\varphi) \check{\boldsymbol{\lambda}} \mathbf{H}^{-1} \boldsymbol{\Xi} \\
\mathbf{H}^{-1}\left(\mathbf{I}_{N}-\check{\boldsymbol{\phi}}_{\pi}-\check{\boldsymbol{\phi}}_{\pi^{d}} \beta\right) & \mathbf{H}^{-1} \boldsymbol{\Xi}
\end{array}\right)
$$

\footnotetext{
${ }^{24}$ Please refer for instance to Horn and Johnson (1985) for a definition of matrix norms.
}

${ }^{25}$ Matrix $\mathbf{A}_{0}^{-1}$ is

$$
\mathbf{A}_{0}^{-1}=\left(\begin{array}{cc}
\check{\boldsymbol{\phi}}_{\pi^{d}}^{-1}\left(\boldsymbol{\Xi}+\check{\boldsymbol{\phi}}_{x}\right) \mathbf{H}^{-1} \check{\boldsymbol{\phi}}_{\pi^{d}} & (1+\varphi) \check{\boldsymbol{\lambda}} \mathbf{H}^{-1} \\
-\mathbf{H}^{-1} \check{\boldsymbol{\phi}}_{\pi^{d}} & \mathbf{H}^{-1}
\end{array}\right) .
$$


where $\mathbf{H}=\check{\boldsymbol{\phi}}_{\pi} \boldsymbol{\Psi}+\mathbf{I}_{N}-\check{\boldsymbol{\phi}}_{\pi}+\check{\boldsymbol{\phi}}_{x}+(1+\varphi) \check{\boldsymbol{\lambda}}_{\pi^{d}}$. Norm of each of the four submatrices of $\mathbf{A}$ is computed below. First note that

$$
\begin{aligned}
\|\boldsymbol{\Xi}\|_{r} & =\max _{i \in\{1, \ldots, N\}}\left(\sum_{j=1, j \neq i}^{N}\left|\phi_{\pi i} \psi_{i j}\right|+\left|1-\phi_{\pi i}\left(1-\psi_{i i}\right)\right|\right)=1 \text { for } \phi_{\pi i} \in\left[0, \frac{1}{1-\psi_{i i}}\right], \\
\left\|\check{\boldsymbol{\phi}}_{\pi^{d}}\right\|_{r} & =\max _{i \in\{1, \ldots, N\}}\left|\phi_{\pi^{d} i}\right| \equiv \bar{\phi}_{\pi^{d}},\left\|\check{\boldsymbol{\phi}}_{\pi}\right\|_{r}=\max _{i \in\{1, \ldots, N\}}\left|\phi_{\pi i}\right| \equiv \bar{\phi}_{\pi}, \\
\left\|\check{\boldsymbol{\phi}}_{x}\right\|_{r} & =\max _{i \in\{1, \ldots, N\}}\left|\phi_{x i}\right|=\bar{\phi}_{x},\|\boldsymbol{\Psi}\|_{r}=1 .
\end{aligned}
$$

$\left\|\mathbf{H}^{-1}\right\|_{r}$ is bounded above by $\left\|\check{\mathbf{H}}^{-1}\right\|_{r}+\left\|\check{\mathbf{H}}^{-1}-(\check{\mathbf{H}}+\mathbf{E})^{-1}\right\|_{r}$ where $\mathbf{H}=\check{\mathbf{H}}+\mathbf{E}, \check{\mathbf{H}}$ is diagonal matrix and diagonal elements of 'perturbation' matrix $\mathbf{E}$ are zeros. Using the upper bound on the relative error made in computing the inverse (cf equation 5.8.2 of Horn and Johnson (1985)),

$$
\left\|\mathbf{H}^{-1}\right\|_{r} \leq\left\|\check{\mathbf{H}}^{-1}\right\|_{r}+\frac{\left\|\check{\mathbf{H}}^{-1} \mathbf{E}\right\|_{r}}{1-\left\|\check{\mathbf{H}}^{-1} \mathbf{E}\right\|_{r}}\left\|\check{\mathbf{H}}^{-1}\right\|_{r}=\frac{\left\|\check{\mathbf{H}}^{-1}\right\|_{r}}{1-\left\|\check{\mathbf{H}}^{-1} \mathbf{E}\right\|_{r}} \leq \frac{\left\|\check{\mathbf{H}}^{-1}\right\|_{r}}{1-\left\|\check{\mathbf{H}}^{-1}\right\|_{r}\|\mathbf{E}\|_{r}}=\frac{1}{\underline{h}-\bar{m}}
$$

where

$$
\begin{aligned}
\left\|\check{\mathbf{H}}^{-1}\right\|_{r} & =\frac{1}{\underline{h}}, \underline{h} \equiv \min _{i \in\{1, \ldots, N\}}\left|\phi_{\pi i} \psi_{i i}+1-\phi_{\pi i}+\phi_{x i}+(1+\varphi) \lambda_{i} \phi_{\pi^{d} i}\right|, \\
\|\mathbf{E}\|_{r} & =\max _{i \in\{1, \ldots, N\}}\left|\phi_{\pi i}\left(1-\psi_{i i}\right)\right|=\bar{m} \leq \bar{\phi}_{\pi}(1-\underline{k}), \underline{k} \equiv \min _{i \in\{1, \ldots, N\}} \psi_{i i},
\end{aligned}
$$

provided that $\varrho\left(\check{\mathbf{H}}^{-1} \mathbf{E}\right)<1$. This condition is satisfied if $\underline{h}>\bar{m}$. Using these results, we can compute the following upper bounds on the norms of the submatrices of matrix $\mathbf{A}$.

$$
\begin{gathered}
\left\|\beta \check{\boldsymbol{\phi}}_{\pi^{d}}^{-1}\left(\boldsymbol{\Xi}+\check{\boldsymbol{\phi}}_{x}\right) \mathbf{H}^{-1} \check{\boldsymbol{\phi}}_{\pi^{d}}+(1+\varphi) \check{\boldsymbol{\lambda}} \mathbf{H}^{-1}\left(\mathbf{I}_{N}-\check{\boldsymbol{\phi}}_{\pi}\right)\right\|_{r}+\left\|(1+\varphi) \check{\boldsymbol{\lambda}} \mathbf{H}^{-1} \boldsymbol{\Xi}\right\|_{r} \leq \frac{v_{2} \bar{\phi}_{\pi^{d}}+\left(1+v_{3}\right)(1+\varphi) \bar{\lambda}}{\underline{h}-\bar{m}}, \\
\left\|\mathbf{H}^{-1}\left(\mathbf{I}_{N}-\check{\boldsymbol{\phi}}_{\pi}-\check{\boldsymbol{\phi}}_{\pi^{d}} \beta\right)\right\|_{r}+\left\|\mathbf{H}^{-1} \boldsymbol{\Xi}\right\|_{r} \leq \frac{v_{1}+1}{\underline{h}-\bar{m}}
\end{gathered}
$$

where

$$
\begin{aligned}
v_{1} & \equiv \max _{i \in\{1, \ldots, N\}}\left|1-\phi_{\pi i}-\phi_{\pi_{i}} \beta\right| \\
v_{2} & \equiv \max _{i \in\{1, \ldots, N\}} \beta\left\{\left|\frac{\phi_{\pi i}\left(1-\psi_{i i}\right)}{\phi_{\pi^{d}}}\right|+\left|\frac{1-\phi_{\pi i}\left(1-\psi_{i i}\right)+\phi_{x i}}{\phi_{\pi^{d}}}\right|\right\}, \\
v_{3} & \equiv \max _{i \in\{1, \ldots, N\}}\left|1-\phi_{\pi i}\right|, \\
\bar{\lambda} & \equiv \max _{i \in\{1, \ldots, N\}}\left|\lambda_{i}\right| .
\end{aligned}
$$

The conditions of Lemma 1 readily follows. This completes the proof. Examples of monetary policy rules that deliver determinate equilibrium are provided in Section 2.2.

\section{B.3. Proof of Theorem 2.}

Proof. Assumption A5-(i) and A5-(iii) imply

$$
\exists \delta>0, \exists N_{1} \in \mathbb{N} ; \forall N>N_{1}: \varrho\left(\mathbf{A}_{N}\right)<1-\delta .
$$

Since $\varrho\left(\mathbf{A}_{N}\right)<1-\delta$ for any $N>N_{1}$ then the equilibrium is determinate and the solution to the system of equations (2.7) is

$$
\underset{2 N \times 1}{\mathbf{z}_{N} t}=\underset{2 N \times 2 N}{\mathbf{C}_{N}} \underset{2 N \times 1}{\boldsymbol{\varepsilon}_{N} t}
$$

where subscript $N$ is used to denote the number of countries in the world economy. $\lim _{N \rightarrow \infty} \mathbf{z}_{N}$ is not well defined since the dimension of vector $\mathbf{z}_{N} t$ changes with $N$. However the limit of $k$-th element of vector $\mathbf{z}_{N}$ (denoted by $z_{N k t}$ ), where $k=i$ or $k=i+N$, $i$ is fixed, is well defined:

$$
\lim _{N \rightarrow \infty} z_{N} k t=\lim _{N \rightarrow \infty} \sum_{j=1}^{2 N} c_{N k j} \varepsilon_{N} j t
$$


where $(k, j)$-th element of matrix $\mathbf{C}_{N}$ is denoted by $c_{N k j}$ and similarly $\varepsilon_{N j t}$ denotes element $j$ of vector $\varepsilon_{N} t$. Now the focus is on the order of magnitude of elements $\left\{c_{N} k j\right\}_{j=1}^{2 N}$. Matrix $\mathbf{C}_{N}$ is given by

$$
\mathbf{C}_{N}=\sum_{k=1}^{\infty} \mathbf{A}_{N}^{k} \mathbf{A}_{N} 2 \mathbf{R}_{N}^{k}
$$

where

$$
\mathbf{A}_{N}{ }_{2} \mathbf{R}_{N}^{k}=\left(\begin{array}{cc}
\mathbf{0} & \mathbf{0} \\
-\check{\boldsymbol{\rho}}_{N r}^{k} & -\mathbf{\Xi}_{N}\left(\mathbf{I}_{N}-\check{\boldsymbol{\rho}}_{N^{a}}\right) \check{\boldsymbol{\rho}}_{N a}^{k}
\end{array}\right) .
$$

Recall that $\boldsymbol{\Xi}_{N}=\check{\boldsymbol{\phi}}_{N \pi} \mathbf{\Psi}_{N}+\mathbf{I}_{N}-\check{\boldsymbol{\phi}}_{N \pi}$. Noting that coefficients $\left\{\phi_{\pi i}\right\}$ are bounded in $N$, Assumptions A1-A2 imply

$$
\left[-\mathbf{\Xi}_{N}\left(\mathbf{I}_{N}-\check{\boldsymbol{\rho}}_{N} a\right) \check{\boldsymbol{\rho}}_{N a}^{k}\right]_{i j}=\left\{\begin{array}{cc}
\rho_{a j}^{k} O(1) & i=j \\
\rho_{a j}^{k} O\left(N^{-1}\right) & i \neq j
\end{array} .\right.
$$

Choose any $0<\delta_{1}<\delta$ and let $\widetilde{\mathbf{A}}_{N} \equiv\left(\varrho\left(\mathbf{A}_{N}\right)+\delta_{1}\right)^{-1} \mathbf{A}_{N}$. Since $\varrho\left(\widetilde{\mathbf{A}}_{N}\right)<1 \forall N>N_{1}$ then $\lim _{k \rightarrow \infty} \widetilde{\mathbf{A}}_{N}^{k}=0$ and therefore (for each fixed $N>N_{1}$ ) the sequence of elements $\left\{\widetilde{a}_{N}^{(k)}\right\}_{k=1}^{\infty}$ is bounded and its maximum exists, where $\widetilde{a}_{N^{i j}}^{(k)}$ denotes element $(i, j)$ of $\operatorname{matrix} \widetilde{\mathbf{A}}_{N}^{k}$. Let

$$
l_{N i j} \equiv \max _{k \in \mathbb{N}} \widetilde{a}_{N i j}^{(k)} \text {. }
$$

Assumption A5-(ii), solution (B.16) for matrix $\mathbf{A}_{N}$ and Lemma 4 imply

$$
l_{N i j}=\left\{\begin{array}{cc}
O(1) & i=j, \text { or } i=j+N, \text { or } i+N=j \\
O\left(N^{-1}\right) & \text { elsewhere }
\end{array} .\right.
$$

Absolute value of the $(i, j)$-th element of matrix $\mathbf{A}_{N}^{k}$ is therefore bounded by

$$
\left|\left[\mathbf{A}_{N}^{k}\right]_{i j}\right| \leq l_{N i j}\left(\varrho\left(\mathbf{A}_{N}\right)+\delta_{1}\right)^{k}
$$

Partition $2 N \times 2 N$ matrix $\mathbf{C}_{N}$ into the following four $N \times N$-dimensional submatrices

$$
\mathbf{C}_{N}=\left(\begin{array}{ll}
\mathbf{C}_{N}, 1 & \mathbf{C}_{N}, 2 \\
\mathbf{C}_{N}, 3 & \mathbf{C}_{N}, 4
\end{array}\right)
$$

Equations (B.17)-(B.19) and Lemma 4 imply that $(i, j)$-th element of each of the 4 submatrices of matrix $\mathbf{C}_{N}$ is bounded by

$$
\left|\left[\mathbf{C}_{N}, k\right]_{i j}\right| \leq\left\{\begin{array}{cc}
O(1) & i=j \\
O\left(N^{-1}\right) & i \neq j
\end{array}\right.
$$

since $\varrho\left(\mathbf{A}_{N}\right)+\delta_{1}<1$ and $\left|\sup _{i \in \mathbb{N}}\left\{\rho_{r i}, \rho_{a i}\right\}\right|<1$. Notice that Lemma 4 also implies that elements of matrix $\mathbf{C}_{N}$ that are not $o(1)$ are function of $O(1)$ but not $o(1)$ elements of matrices $\mathbf{A}_{N}, \mathbf{A}_{2, N}, \mathbf{R}_{N}$ only. Recall that

$$
\begin{aligned}
& \pi_{N i t}^{d}=\sum_{j=1}^{N}\left(c_{N i j} \varepsilon_{N} r j t+c_{N} i, j+N \varepsilon_{N} a j t\right) \\
& x_{N i t}=\sum_{j=1}^{N}\left(c_{N} i+N, j \varepsilon_{N} r j t+c_{N i+N, j+N} \varepsilon_{N} a j t\right)
\end{aligned}
$$

Using equations (B.20), it follows from Lemma 5

$$
\begin{aligned}
& \pi_{N i t}^{d}-\left(\lim _{N \rightarrow \infty} c_{N} i \varepsilon_{N} r i t+\lim _{N \rightarrow \infty} c_{N i, i+N} \varepsilon_{N} \text { ait }\right) \stackrel{q . m .}{\rightarrow} 0, \\
& x_{N i t}-\left(\lim _{N \rightarrow \infty} c_{N i+N, i} \varepsilon_{N} \text { rit }+\lim _{N \rightarrow \infty} c_{N i+N, i+N} \varepsilon_{N} \text { ait }\right) \stackrel{q . m .}{\rightarrow} 0,
\end{aligned}
$$

as $N \rightarrow \infty$. Since $\lim _{N \rightarrow \infty} c_{N i j}$ and $\lim _{N \rightarrow \infty} c_{N} i+N, j$ for $j \in\{i, i+N\}$ is not a function of off-diagonal elements of matrix $\boldsymbol{\Psi}$ (Lemma 4 ), it is possible to equivalently write the system of equation (2.7), when $N \rightarrow \infty$, as individual systems of country-specific equations given in Theorem 2, namely 


$$
\begin{aligned}
& \mathbf{G}_{0 i} \mathbf{z}_{i t}=\mathbf{G}_{1 i} \mathrm{E}_{t} \mathbf{z}_{i, t+1}+\mathbf{G}_{2 i} \boldsymbol{\varepsilon}_{i t} \quad \text { for } \forall i=1,2, \ldots, \\
& \text { where } \underset{2 \times 1}{\mathbf{z}_{i t}}=\left(\begin{array}{c}
\pi_{i t} \\
x_{i t}
\end{array}\right), \underset{2 \times 2}{\mathbf{G}_{0 i}}=\left(\begin{array}{cc}
1 & -(1+\varphi) \lambda_{i} \\
\phi_{\pi^{d} i} & 1-\phi_{\pi i}\left(1-k_{i}\right)+\phi_{x i}
\end{array}\right), \underset{2 \times 2}{\mathbf{G}_{1 i}}=\left(\begin{array}{cc}
\beta & 0 \\
1-\phi_{\pi i} & 1-\phi_{\pi i}\left(1-k_{i}\right)
\end{array}\right) \text {, } \\
& \mathbf{G}_{2 i}=\left(\begin{array}{cc}
0 & 0 \\
-1 & \left(\rho_{a i}-1\right)\left(1-\phi_{\pi i}\left(1-k_{i}\right)\right)
\end{array}\right), \boldsymbol{\varepsilon}_{i t}=\left(\begin{array}{c}
\varepsilon_{r i t} \\
\varepsilon_{a i t}
\end{array}\right) \text {. }
\end{aligned}
$$

\section{B.4. Proof of Theorem 3.}

Proof. Following the proof of Theorem 2 we have:

$$
\begin{aligned}
& \pi_{N i t}^{d}=\sum_{j=1}^{N}\left(c_{N i j} \varepsilon_{r_{N} j t}+c_{N i, j+N} \varepsilon_{a_{N} j t}\right), \\
& x_{N i t}=\sum_{j=1}^{N}\left(c_{N i+N, j} \varepsilon_{r_{N} j t}+c_{N} i+N, j+N\right. \\
&\left.\varepsilon_{a_{N} j t}\right),
\end{aligned}
$$

where the only coefficients of the four submatrices $\mathbf{C}_{N}, k, k=1, . ., 4$ of the matrix $\mathbf{C}_{N}$ that could have nonzero limit are, using again Lemma 4 ,

(1) coefficients on the diagonal,

(2) $\left[\mathbf{C}_{N}, k\right]_{i j}$, where $k=1, . ., 4$, and $i, j \in\{1, . ., N\}$ such that $\left.i\right) \lim _{N \rightarrow \infty} \psi_{N} i j \neq 0$ or ii) $\exists$ a directed path $\gamma_{i j}$ in the directed graph $\Gamma\left(\mathbf{Z}_{\Psi}\right)$ connecting nodes $T_{i}$ and $T_{j}$ of $\Gamma\left(\mathbf{Z}_{\Psi}\right)$.

In each row of the four submatrices $\mathbf{C}_{N}, k, k=1, . ., 4$, coefficients are of order $O\left(N^{-1}\right)$ with the exception of a finite number of countries. Using Lemma 5 completes the proof.

\section{B.5. Proof of Theorem 4.}

Proof. Proof of Theorem 4 closely follows proof of Theorem 2, but this time Lemma 6 is used instead of Lemma 4. Particularly, we have:

$$
\left[-\mathbf{\Xi}_{N}\left(\mathbf{I}_{N}-\check{\boldsymbol{\rho}}_{N a}\right) \check{\boldsymbol{\rho}}_{N}^{k}\right]_{i j}=\left\{\begin{array}{cl}
\rho_{a j}^{k} O(1) & i=j \vee j \in \mathcal{K} \\
\rho_{a j}^{k} O\left(N^{-1}\right) & i \neq j \wedge j \notin \mathcal{K}
\end{array}\right.
$$

and

$$
l_{N i j}=\left\{\begin{array}{c}
O(1) \\
O\left(N^{-1}\right)
\end{array} \quad i=j, \text { or } i=j+N, \text { or } i+N=j, \text { or } j \in \mathcal{K}, \text { or } j-N \in \mathcal{K}\right.
$$

where recall that $l_{N i j} \equiv \max \left\{\left|\widetilde{a}_{N i j}^{(k)}\right|\right\}_{N=1}^{\infty}, \widetilde{a}_{N i j}^{(k)}$ is element $(i, j)$ of matrix $\widetilde{\mathbf{A}}_{N}^{k}, \widetilde{\mathbf{A}}_{N} \equiv$ $\left(\varrho\left(\mathbf{A}_{N}\right)+\delta_{1}\right)^{-1} \mathbf{A}_{N}, 0<\delta_{1}<\delta$, and $\delta$ was chosen such that $\varrho\left(\mathbf{A}_{N}\right)<1-\delta$, which can be done for $\forall N>N_{0}, N_{0}$ being large enough (this is due to Assumptions A5- $(i)$ and A5-(iii)). Absolute value of the $(i, j)$-th element of matrix $\mathbf{A}_{N}^{k}$ is therefore bounded by

$$
\left|\widetilde{a}_{N i j}^{(k)}\right| \leq l_{N i j}\left(\varrho\left(\mathbf{A}_{N}\right)+\delta_{1}\right)^{k}
$$

Equations (B.21)-(B.23) and Lemma 6 imply that the element $(i, j)$ of each of the $4 N \times N$ dimensional submatrices of matrix $\mathbf{C}_{N}$ is bounded by

$$
\left|\left[\mathbf{C}_{N}\right]_{i j}\right| \leq\left\{\begin{array}{cl}
O(1) & i=j \vee j \in \mathcal{K} \\
O\left(N^{-1}\right) & i \neq j \wedge j \notin \mathcal{K}
\end{array},\right.
$$


since $\varrho\left(\mathbf{A}_{N}\right)+\delta_{1}<1$ and $\left|\sup _{i \in \mathbb{N}}\left\{\rho_{r i}, \rho_{a i}\right\}\right|<1$. Hence as $N \rightarrow \infty$, the solution converges for $i \in \mathcal{K}$ to (using Lemma 5 ):

$$
\begin{aligned}
& \pi_{N i t}^{d}-\left(\sum_{j \in \mathcal{K}} \lim _{N \rightarrow \infty} c_{N} i j \varepsilon_{N} r j t+\sum_{j \in \mathcal{K}} \lim _{N \rightarrow \infty} c_{N} i, j+N \varepsilon_{N} a j t\right) \stackrel{q . m .}{\rightarrow} 0, \\
& x_{N} i t-\left(\sum_{j \in \mathcal{K}} \lim _{N \rightarrow \infty} c_{N} i+N, j \varepsilon_{N} r j t+\sum_{j \in \mathcal{K}} \lim _{N \rightarrow \infty} c_{N} i+N, j+N \varepsilon_{N} a j t\right) \stackrel{q . m .}{\rightarrow} 0 .
\end{aligned}
$$

For $i \notin \mathcal{K}$ :

$$
\begin{gathered}
\pi_{N i t}^{d}-\sum_{j \in \mathcal{K}} \lim _{N \rightarrow \infty} c_{N i j} \varepsilon_{N} r j t-\sum_{j \in \mathcal{K}} \lim _{N \rightarrow \infty} c_{N} i, j+N \varepsilon_{N} \text { ajt }- \\
-\lim _{N \rightarrow \infty} c_{N} i i \varepsilon_{N} r i t-\lim _{N \rightarrow \infty} c_{N} i, i+N \varepsilon_{N} a i t \stackrel{q . m .}{\rightarrow} 0, \\
x_{N} i t-\sum_{j \in \mathcal{K}} \lim _{N \rightarrow \infty} c_{N} i+N, j \varepsilon_{N} r j t-\sum_{j \in \mathcal{K}} \lim _{N \rightarrow \infty} c_{N} i+N, j+N \varepsilon_{N} a j t \\
\quad-\lim _{N \rightarrow \infty} c_{N} i+N, i \varepsilon_{N} r i t-\lim _{N \rightarrow \infty} c_{N} i+N, i+N \varepsilon_{N} a i t \stackrel{q . m .}{\rightarrow} 0 .
\end{gathered}
$$

Proof of Lemma 6 implies that coefficients $\left\{c_{N i j}, c_{N} i, j+N\right\}$ and $\left\{c_{N i+N, j}, c_{N i+N, j+N}\right\}$ in the expressions above have nonzero limit in general. This completes the proof.

\section{Appendix C. Construction of Export and Import Share Matrices, Data Sources and Estimation of Parameter $\widehat{\rho}_{a}$}

Two datasets are employed: IMF DOTS database featuring data on foreign trade and IMF IFS database featuring nominal and real GDP data.

Export and import share matrices are constructed as follows. Recall that elements of export share matrix $\Upsilon$ are given by equation (A.31). Nominator $P_{j i t} C_{j i t} \mathcal{P}_{j} E_{i t}$, that is the nominal value of the exported goods from country $i$ to country $j$ in the numeraire currency, is directly approximated by the observed exports, $E X_{i j t}$. Denominator $P_{i t}^{d} Y_{i t} E_{j t}$, that is nominal GDP in the numeraire currency, is directly taken from IMF IFS database. Elements of the import share matrix satisfy:

$$
\psi_{i j}=\frac{P_{i j t} C_{i j t} \frac{1}{E_{i t}}}{P_{i t} C_{i t} \frac{1}{E_{i t}}} .
$$

$P_{i j t} C_{i j t} / E_{i t}$ represents nominal value goods imported from country $j$ to country $i$ in period $t$, which is proxied by the observed nominal imports. Nominal consumption in the numeraire currency $P_{i t} C_{i t} \frac{1}{E_{i t}}$ is proxied by nominal GDP. Averages of the data during the period 2001-2003 are used to compute import share matrix $\boldsymbol{\Psi}$, export share matrix $\Upsilon$ as well as foreign trade share matrix in the text. One additional adjustment has been made in the case of $\boldsymbol{\Psi}$, and $\boldsymbol{\Upsilon}$. Since nominal value of aggregate imports and/or exports exceeds the GDP in few economies, foreign trade series were scaled down so that the $\psi_{i i}$ and $\chi_{i i}$ would not be lower than $40 \%$. Note that due to the data limitations, constructed matrices $\boldsymbol{\Psi}$ and $\boldsymbol{\Upsilon}$ are only imperfect proxies. One particular disadvantage of using IMF DOTS data is that reported imports to country $i$ from country $j$ are not necessarily produced in country $j$ and consumed in country $i$. Thus the importance of economies such as Singapore, or Thailand are likely to be overestimated.

C.1. Estimation of parameter $\widehat{\rho}_{a}$. Homogenous persistence of idiosyncratic innovations to productivity process, parameter $\rho_{a}$, is calculated as an average of the countryspecific parameters $\widehat{\rho}_{a i}$, estimated using following OLS regressions

$$
\Delta a_{i t}=\delta_{0}+\rho_{a i} \Delta a_{i, t-1}+\delta_{a 1} \Delta a_{i t}^{*}+\delta_{a 2} \Delta a_{i, t-1}^{*}+v_{i t}, i=1,2, \ldots, 172 .
$$

This estimation strategy allows for a general $I(1)$ unobserved common factor $f_{t}$ and weakly dependent innovations, see Chudik and Pesaran (2010). $a_{i t}$ is proxied by real GDP per capita. Weights used to construct $a_{i t}^{*}$ are arithmetic averages, with the weight for domestic economy set to 0. Annual data spanning period 1965-2005 for 172 countries 
is used. Histogram of individual estimates $\widehat{\rho}_{a i}$ is presented in Figure 7. Average value $\widehat{\rho}_{a}=\frac{1}{172} \sum_{i=1}^{172} \widehat{\rho}_{a i}=22 \%$. CD test by Pesaran (2004) for residuals without cross-sectional averages in the regression is $C D_{1}=44.98$, well above the critical value for the null of no cross-dependence. Once star variables are included, the value of CD test dropped substantially to $C D_{2}=0.42$, statistically insignificant at $10 \%$ nominal level. We also estimate $\widehat{\rho}_{a i}$ with the common factor(s) proxied by the method of principal components, allowing for up to 10 common factors. Averages of the country-specific estimates lies in the range $20 \%-30 \%$.

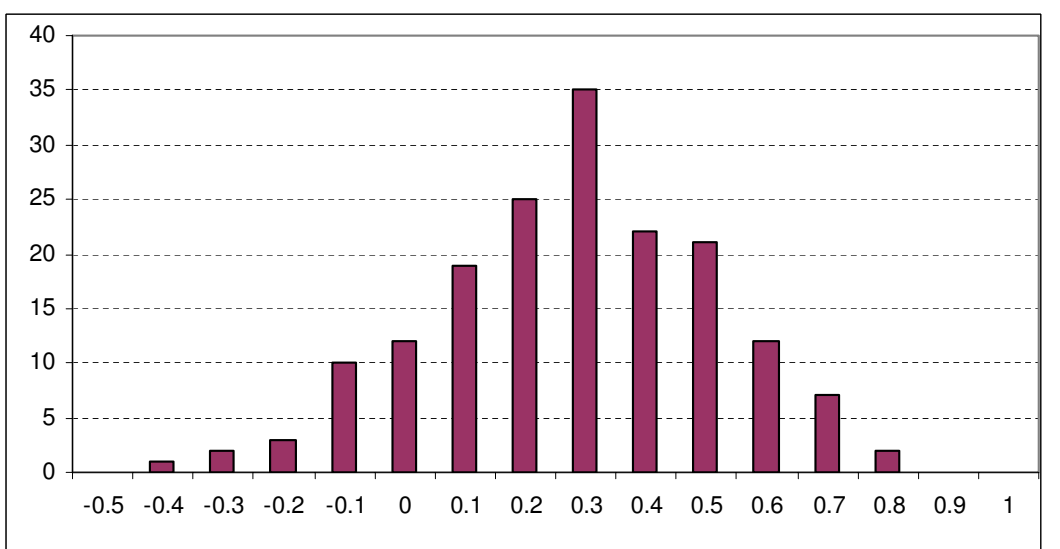

Figure 7: Histogram of estimated country-specific coefficients $\widehat{\rho}_{a i}$ in the regressions for produtivity process.

\begin{tabular}{|l|l|l|}
\hline \hline Parameter & Value & Brief Description \\
\hline$\phi_{\pi i}$ & 1 & Parameters in the monetary policy functions \\
$\phi_{\pi^{d} i}$ & 3 & $r_{i t}=\mathrm{E}_{t} \pi_{i, t+1}+3 \pi_{i t}^{d}+0.3 x_{i t}-\ln \beta+\varepsilon_{r i t}$ \\
$\phi_{x i}$ & 0.3 & Discount factor. \\
\hline$\beta$ & 0.98 & Firms cannot adjust prices with probability $\delta_{i}$. \\
\hline$\delta_{i}\left(\lambda_{i}\right)$ & $27 \%$ (implying $\left.\lambda_{i}=27 \%\right)$ & $\mathrm{AR}(1)$ coefficient, $\varepsilon_{a i t}=\rho_{a i} \varepsilon_{a i, t-1}+\zeta_{\varepsilon_{a} i t}$ \\
\hline$\rho_{a i}$ & 0.22 & $\mathrm{AR}(1)$ coefficient, $\varepsilon_{r i t}=\rho_{r i} \varepsilon_{r i, t-1}+\zeta_{r i t}$ \\
\hline$\rho_{r i}$ & 0.5 & \\
\hline$\varphi$ & 3 & \\
\hline \multicolumn{2}{|l|}{ Size of shock: (Negative) 2 standard deviations. } \\
\hline \multicolumn{2}{|l|}{ Number of replications $R=10000}$.
\end{tabular}

TABLE 2: Summary of parameters for the simulation exercise 


\section{References}

Anderson, J. and V. Wincoop (2003). Gravity with gravitas: Solution to the border puzzle. American Economic Review 93, 170-192.

Baier, S. and J. Bergstrand (2001). The growth of world trade: Tariffs, transport costs, and income similarity. Journal of International Economics 53, 1-27.

Benigno, G. and P. Benigno (2003). Price stability in open economies. Review of Economic Studies 70(4), 743-764.

Binder, M. and M. H. Pesaran (1997). Multivariate linear rational expectations models: characterization of the nature of the solutions and their fully recursive computation. Econometric Theory 13, 877-888.

Calvo, G. (1983). Staggered prices in a utility maximizing framework. Journal of Monetary Economics 12, 383-398.

Canova, F. and M. Ciccarelli (2004). Forecasting and turning point prediction in a Bayesian panel VAR model. Journal of Econometrics 120, 327-359.

Canova, F. and M. Ciccarelli (2009). Estimating multi-country VAR models. International Economic Review 50(3), 929-959.

Chamberlain, G. (1983). Funds, factors, and diversification in arbitrage pricing models. Econometrica 51, 1281-1304.

Chamberlain, G. and M. Rothschild (1983). Arbitrage, factor structure and meanvariance analysis in large asset market. Econometrica 51, 1305-1324.

Chari, V. V., P. Kehoe, and E. McGrattan (2002). Can sticky price models generate persistent real exchange rates? Review of Economic Studies 69(3), 533-563.

Chudik, A. (2008). Global Macroeconomic Modelling. Ph. D. thesis, Trinity College, University of Cambridge.

Chudik, A. and M. H. Pesaran (2010). Infinite dimensional VARs and factor models. Forthcoming in Journal of Econometrics.

Chudik, A., M. H. Pesaran, and E. Tosetti (2010). Weak and strong cross section dependence and estimation of large panels. Forthcomming in the Econometrics Journal.

Corsetti, G. and P. Pesenti (2001). Welfare and macroeconomic interdependence. Quarterly Journal of Economics 116(2, 421-445.

Del Negro, M. and F. Schorfheide (2004). Priors from general equilibrium models for VARs. International Economic Review 45, 643-673.

Gali, J. and T. Monacelli (2005). Monetary policy and exchange rate volatility in a small open economy. Review of Economic Studies 72, 707-734.

Horn, R. A. and C. R. Johnson (1985). Matrix Analysis. Cambridge University Press.

Kollmann, R. (2001). The exchange rate in a dynamic-optimizing business cycle model with nominal rigidities: A quantitative investigation. Journal of International Economics 55, 243-262.

Pesaran, M. H. (2004). General diagnostic tests for cross section dependence in panels. Cambridge Working Paper in Economics 0435, June 2004.

Pesaran, M. H. (2006). Estimation and inference in large heterogenous panels with multifactor error structure. Econometrica 74, 967-1012.

Pesaran, M. H. and E. Tosetti (2010). Large panels with common factors and spatial correlation. CESifo Working Paper No. 2103, September 2007, revised January 2010.

Schmitt-Grohe, S. and U. Uribe (2003). Closing small open economy models. Journal of International Economics 61, 163-185.

Woodford, M. (2003). Interest 8 Prices: Foundations of a theory of monetary policy. Princeton University Press. 